\title{
BMJ Open How effective are brief interventions in reducing alcohol consumption: do the setting, practitioner group and content matter? Findings from a systematic review and metaregression analysis
}

\author{
Lucy Platt, ${ }^{1}$ G J Melendez-Torres, ${ }^{2}$ Amy O'Donnell, ${ }^{3}$ Jennifer Bradley, ${ }^{3}$ \\ Dorothy Newbury-Birch, ${ }^{4}$ Eileen Kaner, ${ }^{3}$ Charlotte Ashton ${ }^{5}$
}

To cite: Platt L, MelendezTorres GJ, O'Donnell A, et al. How effective are brief interventions in reducing alcohol consumption: do the setting, practitioner group and content matter? Findings from a systematic review and metaregression analysis. BMJ Open 2016;6:e011473.

doi:10.1136/bmjopen-2016011473

- Prepublication history and additional material is available. To view please visit the journal (http://dx.doi.org/ 10.1136/bmjopen-2016011473).

Received 11 February 2016 Revised 15 June 2016 Accepted 14 July 2016

CrossMark

For numbered affiliations see end of article.

Correspondence to Dr Lucy Platt; lucy. platt@Ishtm.ac.uk

\section{ABSTRACT}

Background: While the efficacy and effectiveness of brief interventions for alcohol (ABI) have been demonstrated in primary care, there is weaker evidence in other settings and reviews do not consider differences in content. We conducted a systematic review to measure the effect of $A B I s$ on alcohol consumption and how it differs by the setting, practitioner group and content of intervention.

Methods: We searched MEDLINE, EMBASE, PsycINFO; CINAHL, Social Science Citation Index, Cochrane Library and Global Health up to January 2015 for randomised controlled trials that measured effectiveness of $A B I s$ on alcohol consumption. We grouped outcomes into measures of quantity and frequency indices. We used multilevel meta-analysis to estimate pooled effect sizes and tested for the effect of moderators through a multiparameter Wald test. Stratified analysis of a subset of quantity and frequency outcomes was conducted as a sensitivity check.

Results: 52 trials were included contributing data on 29891 individuals. ABIs reduced the quantity of alcohol consumed by $0.15 \mathrm{SDs}$. While neither the setting nor content appeared to significantly moderate intervention effectiveness, the provider did in some analyses. Interventions delivered by nurses had the most effect in reducing quantity $(d=-0.23$, $95 \% \mathrm{Cl}(-0.33$ to -0.13$))$ but not frequency of alcohol consumption. All content groups had statistically significant mean effects, brief advice was the most effective in reducing quantity consumed $(\mathrm{d}=-0.20,95 \% \mathrm{Cl}(-0.30$ to -0.09$))$. Effects were maintained in the stratified sensitivity analysis at the first and last assessment time.

Conclusions: ABIs play a small but significant role in reducing alcohol consumption. Findings show the positive role of nurses in delivering interventions. The lack of evidence on the impact of content of intervention reinforces advice that services should select the $A B I$ tool that best suits their needs.

\section{Strengths and limitations of this study}

- A key strength of this review is the methodologically innovative approach to the meta-analysis through the use of a multilevel meta-analysis.

- As a second sensitivity analysis we compared the findings from the multilevel model with a stratified analysis focusing on a subset of outcome variables. Findings from the two analyses were comparable.

- Quality assessment criteria were used to assess risk of bias and the majority of studies were at low risk in relation to the randomisation procedure and monitoring of loss to follow-up.

- A large proportion of studies did not provide information on other aspects of the study design including blinding of participants to the intervention, intention-to-treat analysis and blinding to outcome measurements.

- Our review suggested limited effect for interventions delivered in community settings, but relied on a small number of studies across a wide variety of settings.

\section{INTRODUCTION}

Excessive alcohol consumption is a major public health concern, contributing to almost $4 \%$ of deaths worldwide, ${ }^{1}$ ranging from as high as $8 \%$ of deaths among men and women in the USA and Norway to $1.4 \%$ in the UK. ${ }^{2}{ }^{3}$ It is estimated that over 10 million people in the UK alone drink more than the recommended daily units. ${ }^{4}$ Screening to detect individuals drinking alcohol at hazardous or harmful levels and the delivery of a brief intervention on alcohol (ABI) to reduce their consumption have been implemented in primary care settings where their efficacy and effectiveness have been demonstrated. ${ }^{1}$ The content of 
ABIs is varied, but usually focuses on the provision of structured advice, involving an assessment of individual risk with feedback and advice, or brief motivational interviewing that takes a more patient-centred approach, or a combination of both. ${ }^{5}$ Existing systematic reviews have found variability in effect by duration of intervention or number of visits, but this has not taken into account differences in content or provider. ${ }^{6-10}$ Although there is some emerging evidence that motivational interviewing can be more effective than 'traditional' advice (based on a provider-centred definition of a problem) across a range of health behaviours, ${ }^{11}$ this is not conclusive. ${ }^{12}$ Further, while the efficacy and effectiveness of ABIs have been demonstrated in primary care settings ${ }^{13-15}$ the evidence base in health settings beyond primary care is weaker with moderate or no effect found in college ${ }^{1617}$ and community settings. ${ }^{18}$ Some benefits have been observed from a small number of studies in accident and emergency (A\&E) departments, ${ }^{19}{ }^{20}$ as well as in general hospital settings but among mainly male patients. ${ }^{21}{ }^{22}$ Implementation research has shown that contextual factors affecting the routine delivery of ABIs in primary healthcare settings are closely linked to practitioners. However, there has been little research looking at the impact of practitioners on intervention effectiveness outside primary healthcare settings. ${ }^{23} 24$

In England, the Government's Alcohol Strategy calls for the increased implementation of ABIs in primary care and A\&E settings, while targets for implementing ABIs in these settings as well as antenatal clinics have been set by the National Health Service (NHS) Scotland. ${ }^{25} 26$ The National Institute for Health and Care Excellence (NICE) guidance recommends that ABI should be offered opportunistically by a range of relevant practitioners and front-line staff, while also acknowledging that the strength of evidence was clearer in some health settings compared with others. Nevertheless, this guidance flagged the relevance of social care, criminal justice, community and voluntary sector professionals to supporting alcohol risk-reduction work. $^{27}$ This recommendation has been implemented by some public health authorities, rolling out interventions in sexual health clinics and community settings such as criminal justice services, and has also been advocated by global health agencies including the WHO. ${ }^{28}$ Given the international-level, national-level and local-level support for the expansion of ABIs beyond primary care settings, there is an urgent need to understand how a brief intervention process (including setting, provider and content) moderates their effectiveness in order to inform their implementation. ${ }^{5}$ We therefore undertook a systematic review and metaregression to measure the effect of ABIs on alcohol consumption and how the effect differs by setting, provider group and content of intervention.

\section{METHODS}

Search strategy and selection criteria

We followed the PRISMA guidelines on reporting of systematic reviews. ${ }^{29}$ Studies eligible for this review were peer-reviewed randomised controlled trials of ABIs published in English. We included all populations aged 16 years or older but excluded populations with complex health problems, for example, studies of people living with HIV, tuberculosis, hepatitis $\mathrm{C}$ virus or homeless populations where it is difficult to generalise findings to the general population. Similarly we excluded populations seeking help at specialist addiction centres, mental health services or antenatal clinics. We included studies with control groups comprising: treatment as usual; information-only; assessment only; no assessment; or non-intervention, and excluded control groups consisting of other interventions, including other brief interventions such as advice and extended psychological treatments. Brief interventions were defined as person-to-person discussions on alcohol between one and four sessions and not more than 2 hours total intervention time. Computerised interventions tested alone, group interventions and those that target multiple behaviours were excluded. We also excluded studies where no measure of alcohol consumption was reported.

The primary outcome of interest was a quantitative continuous measure of total alcohol consumption within a specified time frame (standard drinks, grams of ethanol or days of drinking) where the standardised mean difference between brief intervention and control group was measured at the time of follow-up.

We searched: MEDLINE; EMBASE; PsycINFO; CINAHL; Social Science Citation Index and Science Citation Index through Web of Science; Cochrane Effective Practice and Organisation of Care Group specialised register; and Global Health between 1966 and 2015. The search was conducted in January 2015. We also scanned citations and contacted experts in the field to minimise selection bias. The search terms used were: 'Brief intervention' OR 'minimal intervention' OR 'early intervention' OR 'cognitive behavioural' OR 'screening' OR 'counselling' OR 'brief advice' OR 'identification' OR 'managed care' or 'motivational interview' AND 'Alcohol drinking' or 'binge drinking' OR 'alcohol consumption' OR 'alcohol units' OR 'alcohol use and misuse' OR 'alcohol intake' OR 'alcohol rate binge drinking' OR 'beer or wine or lager or spirit drinking' AND 'randomized controlled trial' OR 'random allocation' OR 'double blind methods' OR 'clinical trial' OR 'controlled clinical trial' OR 'multi centre studies'. Searches were tailored to the search functionality of each database (see web appendix).

Eligibility assessment was conducted independently by two reviewers. Disagreements between reviewers were resolved by consensus. We selected a list of risk of bias criteria from recommendations in the Cochrane Collaboration Reviewers' Handbook to assess the quality of the trials. ${ }^{30}$ Criteria included methods used to generate the allocation sequence to produce comparable groups and concealment of allocation to determine whether intervention allocations could have been foreseen before or during enrolment; blinding of 
participants and providers to intervention groups; blinding of outcome assessment; incomplete outcome data (including intention-to-treat analysis); and measurement of attrition rate.

Data were extracted from each publication into a database piloted on five studies, independently by GJM-T, LP, $\mathrm{AO}$ and JB without blinding authors' names, study site, intervention or trial results. These researchers jointly reviewed the extracted data and $10 \%$ of studies were double extracted. Data were extracted on characteristics of trial participants, type of interventions (including content, duration, frequency, provider, setting), type of outcome measure, time of assessment and effect estimates.

We extracted continuous outcomes in the units in which they were presented and then converted them into Cohen's $d$ for comparability. When extracting continuous outcomes, we preferred estimates that were analysis of covariance adjusted for baseline score, followed by unadjusted post-test scores and finally repeated measures or 'change score' models. Change score models were reparametrised into a raw score metric using $\mathrm{r}=0.5$, with sensitivity analysis at $r=0.1$ and $r=0.9$. Though past reviews have attempted to convert all measures to 'natural units' such as grams of ethanol, we decided that this was inadvisable because of the large number of trials in this review and because of our goal to include all relevant information, a key benefit of multilevel meta-analysis models.

\section{Data synthesis}

We grouped intervention content into three categories (figure 1). The first was motivational interviewing, including motivational interviewing-style, advice approaches such as FRAMES, motivational enhancement therapy as adapted for Project MATCH (Project MATCH
Research Group, 1998) or brief motivational interviewing. We also identified a second subset of trials that tested specific enhanced interventional protocols for motivational interviewing (eg, drink less) or additions to motivational interviewing (eg, cognitive-behavioural approaches) from other therapeutic modalities and labelled this category motivational interviewing 'plus' (MI plus). A third subset included brief advice approaches, often labelled as such without any additional information.

Intervention providers were grouped into: counsellors (defined as any mental health providers including clinical and research psychologists or clinical social workers); general practitioners (including primary care providers and general physicians); nurses (including research or clinical nurses on secondment); peerdelivered and different providers (but with no fixed provider). Setting of intervention delivery was categorised as: A\&E services; community-based delivery that included a range of non-clinical settings; primary or ambulatory care delivered in clinical settings as outpatient services; hospital inpatient services and university services.

The systematic review protocol was registered on PROSPERO at the University of York (CRD42014014799).

\section{Statistical analyses}

We grouped outcomes hierarchically. We identified an overarching set of outcomes addressing quantity of alcohol consumption, from which we created two subsets of outcomes: (1) amount of alcohol consumed per unit of time; and (2) amount of alcohol consumed per drinking occasion. We also identified an overarching set of outcomes addressing frequency of alcohol consumption, from which we created a subset of outcomes including (1) frequency of any drinking occasion; and (2) frequency of binge drinking occasions.
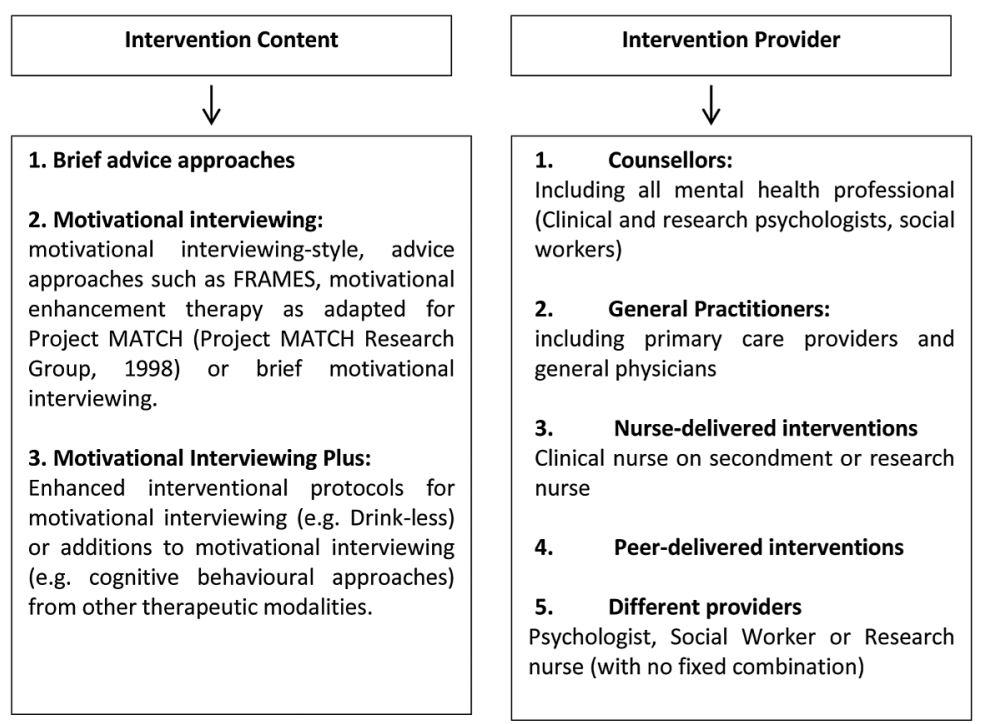

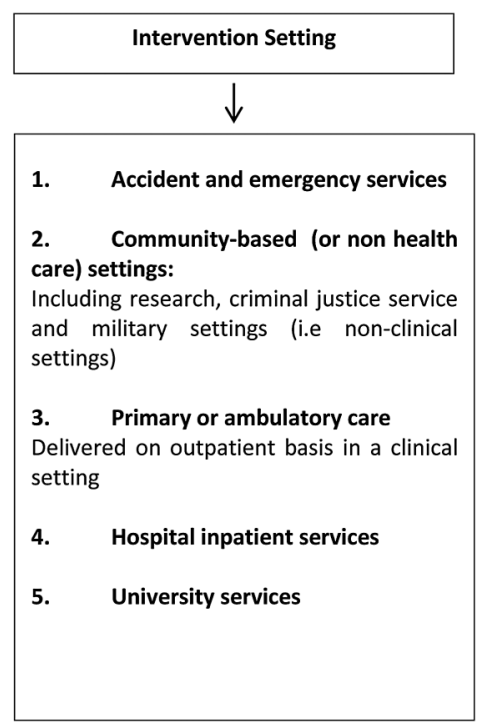

Figure 1 Categories and definitions of interventions by content, provider and setting. 
For each overarching set and subset of outcomes, we specified five models: (1) an unconditional model that included all eligible continuous outcomes; (2) a model that included a grand mean-centred covariate for time of follow-up postbaseline, to address differences in follow-up; (3) a model including where the intervention was initially delivered and time of follow-up; (4) a model including the provider of the intervention and time of follow-up; and (5) a model including the content of the intervention and time of follow-up. To estimate mean effects for all groups simultaneously, we refit models with no intercept. ${ }^{31}$ We used the statistical package metafor, ${ }^{32}$ which implements advanced meta-analysis models, in the R environment for all multilevel analyses.

For our main analysis, we used a multilevel meta-analysis method to estimate the pooled effect sizes. ${ }^{33}$ Models included random effects on the effect size and study levels because of anticipated heterogeneity both within and across studies. Several trials tested different intervention or provider types in the same experiment, but insufficient trials did this to treat intervention as a 'within-trial' covariate. In order to adequately model these two moderators, we split the control groups in two for these trials and treated each intervention-control comparison as a separate trial. This avoided double-counting participants across intervention-control comparisons. Moreover, several studies presented results stratified by group. In our multilevel meta-analyses, we included these in the same cluster. We tested for the effect of our hypothesised moderators by conducting a multiparameter Wald test on provider, setting or content coefficients as appropriate. We additionally examined the residual heterogeneity, measured as $\mathrm{I}^{2}$, between the time-adjusted model and the models including each of the three sets of covariates. We regarded a $p<0.05$ as statistically significant and a $p<0.10$ as marginal, but not significant.

\section{Sensitivity check}

In addition to sensitivity analysis on the correlation used for repeated measures conversion, we estimated a set of metaregressions for each subset of outcomes including one effect size per relevant comparison for each of first and last follow-up in the included trials. We did this by combining intervention and control groups where appropriate, and by selecting effect sizes within studies that used shorter time periods for measurement and timeline follow-back procedures over general frequency/ quantity questionnaires. We also treated non-overlapping subgroups from the same study as separate data points as suggested by Borenstein et $a l^{34}$ Sensitivity analyses were estimated in Stata V.13.1 (Stata Corp 2013) and $\mathrm{R}$ ( $\mathrm{R}$ Core Team. A language and environment for statistical computing. Vienna, Austria: R Foundation for Statistical Computing; 2016). We did not undertake meta-analysis of effect sizes from common time points because these models would have been poorly powered.

\section{RESULTS}

We identified a total of 4551 records from the search of electronic databases and 41 records from key experts. A total of 52 studies met our inclusion criteria, with three studies presenting different outcomes for the same data and therefore considered as one. ${ }^{35-37}$ One study was dropped as it only contained biological outcomes which were not included in the main analyses. ${ }^{38}$ The review and selection process is summarised in figure 2 .

Included studies contributed data for 29891 individuals. Table 1 presents a summary of study characteristics (country, age, sex and sample size) as well as type of intervention (setting, provider and content), key outcomes and time of assessment. Most studies originated from Europe or North America with the exception of three studies from Australia, Taiwan and Thailand. ${ }^{72} 7677$ Almost half $(45 \%)$ of the studies were conducted in the USA and $22 \%$ in the UK.

In total, $68 \%$ of trials were delivered in primary or healthcare settings (hospital or A\&E). Only six studies were conducted in community settings defined as: military; ${ }^{55} \quad 61 \quad 62$ research sites recruiting a sample through a household survey; ${ }^{64}$ and women at risk of alcohol-exposed pregnancy (defined as aged 18-44 years, with ineffective or no use of contraceptives, sexually active in the past 6 months, but not currently pregnant or planning a pregnancy) recruited via the media, in a prison, community health centre and a gynaecology centre; ${ }^{67}$ and one criminal justice setting. ${ }^{87}$ The most common providers included counsellors, who were the sole providers of interventions in $43 \%$ of trials, and physicians who accounted for $24 \%$ of trials. A minority category of different providers (8\%) included a combination of psychologists, social workers or research nurses. Intervention categories were well distributed, though a majority of trials $(47 \%)$ included motivational interviewing alone and 39\% included MI 'plus'. A total of 50 trials reported 275 eligible effect sizes on outcomes measuring quantity of alcohol consumed with a mean follow-up of 9 months. This is summarised in table 2.

The majority $(71 \%)$ of studies were categorised as low risk of bias in relation to randomisation and allocation concealment strategies. In the majority of studies, the process used to assess blinding of participants and providers as well as outcome assessment was unclear. Intention-to-treat analysis was conducted in $47 \%$ of studies and loss to follow-up assessed in the majority $(80 \%)$ of studies. This is summarised in table 3 and risk of bias assessment for all trials is included in the online web appendix table 1 .

\section{Metaregression on combined quantity and frequency outcomes}

Interventions produced a beneficial effect at reducing the quantity of alcohol consumed by $0.15 \mathrm{SDs}-\mathrm{a}$ small but statistically significant effect (see table 4). This effect persisted after controlling for time to follow-up and when examining the subset of outcomes. In unconditional 


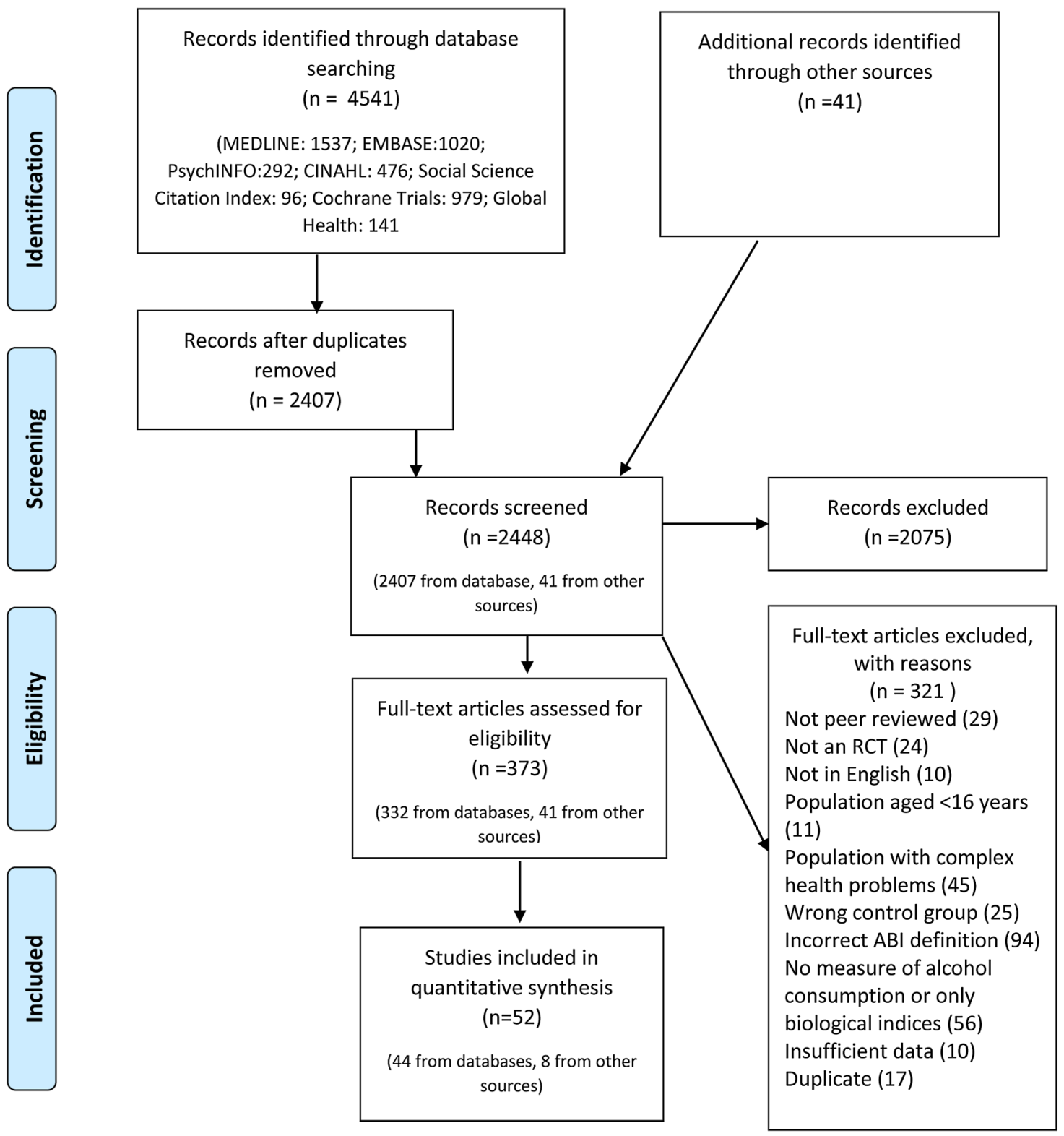

Figure 2 Flow chart of systematic review and study selection. ABI, brief interventions for alcohol; RCT, randomised controlled trial.

models and models controlling for time of follow-up, study-level heterogeneity as measured by $\mathrm{I}^{2}$ (ie, the percentage of variation between effect sizes due to heterogeneity rather than chance) was in the small-to-moderate range $(0-40 \%)$ as defined by the Cochrane Handbook. ${ }^{30}$ Findings were robust to sensitivity analysis on the pre-post correlation in change score models. The mean timeadjusted effect of brief alcohol interventions on frequency of alcohol consumption outcomes was similar in magnitude $(\mathrm{d}=-0.15,95 \%$ CI $(-0.20$ to -0.11$))$, but lower in heterogeneity $\left(\mathrm{I}^{2}=23 \%\right)$, compared with the effect on the quantity of alcohol consumption (table 5). The time-adjusted effect remained statistically significant when limited to the subset of outcomes (frequency of drinking occasions $\mathrm{d}=-0.12$, $95 \%$ CI $(-0.19$ to -0.06$)$ and frequency of binge drinking $\mathrm{d}=-0.17,95 \%$ CI $(-0.23$ to -0.11$)$.

\section{Setting}

For all quantity outcomes the setting of intervention did not appear to fully explain heterogeneity between studies, with residual heterogeneity at $34 \%$ and a statistically marginal but non-significant joint test of moderators $(\mathrm{p}=0.09)$. Interventions conducted in university settings $(d=-0.20,95 \%$ CI $(-0.39$ to -0.09$))$ and in primary or ambulatory care $(-0.20$ to $(-0.27$ to -0.13$))$ appeared to be most effective, with a small but statistically significant effect of the intervention. Interventions delivered in community settings (military, criminal justice, research sites and targeted recruitment) did not appear to be effective $(-0.03(-0.16$ to 0.10$)$; table 4$)$.

For all frequency outcomes, the setting of intervention did not explain heterogeneity (residual $\mathrm{I}^{2}=25 \%$, Wald $\mathrm{p}=0.54$ ). Of subgroups with statistically significant pooled 


\begin{tabular}{|c|c|c|c|c|c|c|c|c|c|}
\hline \multirow[b]{2}{*}{ Author } & \multirow[b]{2}{*}{ Country } & \multirow{2}{*}{$\begin{array}{l}\text { Sample } \\
\mathrm{n}^{\dagger} \text { Per cent of } \mathrm{F}, \\
\text { age (years) }\end{array}$} & \multicolumn{5}{|c|}{ Intervention } & \multicolumn{2}{|l|}{ Outcomes } \\
\hline & & & Setting & Provider & Arm & Content & $\begin{array}{l}\text { Total } \\
\text { minutes } \\
\text { (sessions) }\end{array}$ & $\begin{array}{l}\text { Definition }(Q=\text { quantity, } \\
F=\text { frequency })\end{array}$ & $\begin{array}{l}\text { Time } \\
\text { (months) }\end{array}$ \\
\hline$\overline{\text { Aalto et } a{ }^{\beta 9}}$ & Finland & $118,100 \%, 41$ & GP & $\begin{array}{l}\text { GP/nurse } \\
\text { GP only } \\
\text { NA }\end{array}$ & $\begin{array}{l}1 \\
2 \\
C\end{array}$ & $\begin{array}{l}\mathrm{MI} \\
\mathrm{MI} \\
\mathrm{TAU}\end{array}$ & $\begin{array}{l}70-130(7) \\
30-60(7)\end{array}$ & $\begin{array}{l}\text { Q: amount per week; usual } \\
\text { amount per occasion (g); } \\
\text { F: drinking times per week }\end{array}$ & 36 \\
\hline Aalto et $a{ }^{40}$ & Finland & $296,0 \%, 41$ & GP & $\begin{array}{l}\text { GP/nurse } \\
\text { GP only } \\
\text { NA }\end{array}$ & $\begin{array}{l}1 \\
2 \\
\mathrm{C}\end{array}$ & $\begin{array}{l}\mathrm{Ml} \\
\mathrm{Ml} \\
\mathrm{TAU}\end{array}$ & $\begin{array}{l}70-130(7) \\
30-60(7)\end{array}$ & $\begin{array}{l}\text { Q: grams per week/per occasion } \\
\text { F: drinking times per week }\end{array}$ & 36 \\
\hline Anderson and Scott ${ }^{41}$ & UK & $154,0 \%, 44$ & GP & GP & $\begin{array}{l}1 \\
2\end{array}$ & $\begin{array}{l}\text { BA } \\
\text { TAU }\end{array}$ & $10(1)$ & $\begin{array}{l}\text { Q: breath alcohol (mg/100 } \mathrm{ml} \text { ); } \\
\text { HSQ quantity/frequency and } \\
\text { interview (g/week) }\end{array}$ & 12 \\
\hline Antti-Poika et a ${ }^{42}$ & Finland & $120,0 \%, 39$ & $A \& E$ & Nurse & $\begin{array}{l}1 \\
\mathrm{C}\end{array}$ & $\begin{array}{l}\text { BA } \\
\text { NR }\end{array}$ & NR (1) & $\begin{array}{l}\text { Q: grams of absolute alcohol } \\
\text { during } 1 \text { week period }\end{array}$ & $6(P-I)$ \\
\hline Baer et $a l^{43}$ & USA & $508,55 \%, N R$ & College & Counsellor & 1 & $\begin{array}{l}\text { MI } \\
\text { Screening }\end{array}$ & $\begin{array}{l}\text { Unclear } \\
\text { (NR) }\end{array}$ & $\begin{array}{l}\text { Q: mean drinks per drinking } \\
\text { day; F: drinking days per } \\
\text { average week }\end{array}$ & $24 ; 36$ \\
\hline Beich et $a^{44}$ & Denmark & $6897,62 \%, 36$ & GP & GP & $\begin{array}{l}1 \\
\mathrm{C}\end{array}$ & $\begin{array}{l}\text { Ml plus } \\
\text { Screening }\end{array}$ & $10(1)$ & $\begin{array}{l}\text { Q: usual weekly consumption of } \\
\text { beer, wine and spirits (units/ } \\
\text { week) }\end{array}$ & 12 \\
\hline Bernstein et $a{ }^{45}$ & USA & $835,56 \%, 88 \%>18$ & $A \& E$ & Peer & $\begin{array}{l}1 \\
C\end{array}$ & $\begin{array}{l}\text { MI } \\
\text { Screening }\end{array}$ & Unclear (1) & $\begin{array}{l}\text { Q: maximum drinks per day; } \\
\text { mean drinks per drinking day; } \\
\text { mean drinks per week } \\
\text { F: drinking days per month }\end{array}$ & 3,12 \\
\hline Butler et $a{ }^{46}$ & USA & $114,65 \%, 20$ & College & $\begin{array}{l}\text { Media } \\
\text { Counsellor } \\
\text { NA }\end{array}$ & $\begin{array}{l}1 \\
2 \\
\mathrm{C}\end{array}$ & $\begin{array}{l}\text { BA } \\
\text { MI } \\
\text { Screening }\end{array}$ & $\begin{array}{l}11(1) \\
41(1)\end{array}$ & $\begin{array}{l}\text { Q: standard drinks per week; } \\
\text { F: binge episodes; drinking } \\
\text { occasions; drinking occasions }\end{array}$ & $1(P-I)$ \\
\hline Carey et $a A^{47}$ & USA & $509,65 \%, 19$ & College & Counsellor & $\begin{array}{l}1 \\
2 \\
C\end{array}$ & $\begin{array}{l}\text { MI } \\
\text { MI plus } \\
\text { Screening }\end{array}$ & $\begin{array}{l}65(1) \\
70(1)\end{array}$ & $\begin{array}{l}\text { Q: drinks per drinking day; } \\
\text { F: drinks per week; heavy } \\
\text { drinking frequency }\end{array}$ & 6 or 12 \\
\hline Cherpitel et $a f^{48}$ & Poland & $446,17 \%, 54 \%>30$ & $A \& E$ & Nurse & $\begin{array}{l}1 \\
\mathrm{C} \\
\mathrm{C}\end{array}$ & $\begin{array}{l}\text { Ml plus } \\
\text { Screening } \\
\text { Assessment }\end{array}$ & $15-20(3)$ & $\begin{array}{l}\text { Q: drinks per drinking day; } \\
\text { maximum drinks per occasion } \\
\text { past month; } \\
\text { F: drinking days per week }\end{array}$ & 12 \\
\hline Chick et $a^{49}$ & UK & $156,0 \%, 18-65$ & $A \& E$ & Nurse & $\begin{array}{l}1 \\
2\end{array}$ & $\begin{array}{l}\text { BA } \\
\text { Screening }\end{array}$ & $60(1)$ & $\begin{array}{l}\text { Q: consumption on past week } \\
\text { (units) }\end{array}$ & 12 \\
\hline Cordoba et $a^{50}$ & Spain & $229,0 \%, 36.5$ & GP & GP & $\begin{array}{l}1 \\
\mathrm{C}\end{array}$ & $\begin{array}{l}\text { Branded } \\
\text { Simple } \\
\text { advice }\end{array}$ & $15(1)$ & $\begin{array}{l}\text { Q: alcohol consumption units/ } \\
\text { week }\end{array}$ & 12 \\
\hline Crawford et $a^{51}$ & UK & $599,21 \%, 44$ & $A \& E$ & Nurse & $\begin{array}{l}1 \\
C\end{array}$ & $\begin{array}{l}\text { MI } \\
\text { Information }\end{array}$ & $30(3)$ & $\begin{array}{l}\text { Q: mean units per drinking day; } \\
\text { mean weekly units }\end{array}$ & 6 or 12 \\
\hline
\end{tabular}




\begin{tabular}{|c|c|c|c|c|c|c|c|c|c|}
\hline \multirow[b]{2}{*}{ Author } & \multirow[b]{2}{*}{ Country } & \multirow{2}{*}{$\begin{array}{l}\text { Sample } \\
\mathbf{n}^{\dagger} \text { Per cent of } \mathrm{F} \text {, } \\
\text { age (years) }\end{array}$} & \multicolumn{5}{|l|}{ Intervention } & \multicolumn{2}{|l|}{ Outcomes } \\
\hline & & & Setting & Provider & Arm & Content & $\begin{array}{l}\text { Total } \\
\text { minutes } \\
\text { (sessions) }\end{array}$ & $\begin{array}{l}\text { Definition }(Q=\text { quantity, } \\
F=\text { frequency) }\end{array}$ & $\begin{array}{l}\text { Time } \\
\text { (months) }\end{array}$ \\
\hline Crawford et $a^{F^{2}}$ & UK & $802,54 \%, 27$ & GP & Nurse & $\begin{array}{l}1 \\
\mathrm{C}\end{array}$ & $\begin{array}{l}\text { BA } \\
\text { Information }\end{array}$ & $2-3(1)$ & $\begin{array}{l}\text { Q: mean units on drinking days; } \\
\text { weekly alcohol consumption in } \\
\text { units }\end{array}$ & 6 \\
\hline Curry et $a^{{ }^{33}}$ & USA & $333,35 \%, 47$ & GP & $\begin{array}{l}\text { GP and } \\
\text { counsellor }\end{array}$ & $\begin{array}{l}1 \\
\mathrm{C}\end{array}$ & $\begin{array}{l}\text { MI plus } \\
\text { TAU }\end{array}$ & $47(1)$ & Q: drinks per week & 12 \\
\hline Daeppen et $a^{54}$ & Switzerland & $987,22 \%, 36.7$ & $A \& E$ & Counsellor & $\begin{array}{l}1 \\
\mathrm{C} \\
\mathrm{C}\end{array}$ & $\begin{array}{l}\text { MI } \\
\text { Assessment } \\
\text { Nothing }\end{array}$ & $17(1)$ & $\begin{array}{l}\text { Q: number of drinks per } \\
\text { occasion/past week (past year) } \\
\text { F: number of binge drinking } \\
\text { occasions per month/per week } \\
\text { (past year) }\end{array}$ & 12 \\
\hline Daeppen et $a^{55}$ & Switzerland & $2831,0 \%, 19.9$ & $\begin{array}{l}\text { Community } \\
\text { (military) }\end{array}$ & Counsellor & $\begin{array}{l}1 \\
\mathrm{C}\end{array}$ & $\begin{array}{l}\text { MI } \\
\text { Assessment }\end{array}$ & $15.8(2)$ & $\begin{array}{l}\text { Q: change in drinks per week } \\
\text { F: change in binge drinking } \\
\text { occasions per month }\end{array}$ & 6 \\
\hline Drummond et $a^{56}$ & UK & $1204,35 \%, 34.6$ & $A \& E$ & Counsellor & $\begin{array}{l}1 \\
2 \\
C\end{array}$ & $\begin{array}{l}\text { Branded } \\
\text { MI } \\
\text { Information }\end{array}$ & $\begin{array}{l}20(1) \\
20(1)\end{array}$ & Q: average daily drinks & $6,12(P-I)$ \\
\hline Field et $a^{77}$ & USA & $1439,18 \%, 33$ & $A \& E$ & Counsellor & $\begin{array}{l}1 \\
\mathrm{C}\end{array}$ & $\begin{array}{l}\text { MI } \\
\text { TAU+assess }\end{array}$ & Unclear (1) & $\begin{array}{l}\text { Q: change in: alcohol per week; } \\
\text { maximum amount in a day in } \\
\text { past } 6 \text { months; } \\
\text { F: change in per cent days } \\
\text { heavy drinking }\end{array}$ & 6,12 \\
\hline $\begin{array}{l}\text { Fleming and Manwell; } \\
\text { Manwell et al, } \\
\text { Grossberg et al }\end{array}$ & USA & $\begin{array}{l}774,38 \%, 29 \% 18- \\
30\end{array}$ & GP & $\begin{array}{l}\text { GP and } \\
\text { nurse }\end{array}$ & $\begin{array}{l}1 \\
\mathrm{C}\end{array}$ & $\begin{array}{l}\text { Branded } \\
\text { Information }\end{array}$ & $30(2)$ & $\begin{array}{l}\text { Q: number of drinks in past } \\
7 \text { days } \\
\text { F: number of binge drinking } \\
\text { episodes in past } 30 \text { days (binge } \\
\text { drinking defined as having more } \\
\text { than } 4 \text { drinks per occasion) }\end{array}$ & $\begin{array}{l}6,12,24 \\
36,48 \\
(P-I)\end{array}$ \\
\hline $\begin{array}{l}\text { Fleming and } \\
\text { Manwell }^{58}\end{array}$ & USA & $158,34 \%, 65-75$ & GP & GP/nurse & $\begin{array}{l}1 \\
\mathrm{C}\end{array}$ & $\begin{array}{l}\text { Branded } \\
\text { Information }\end{array}$ & $30(2)$ & $\begin{array}{l}\text { Q: number of drinks in past } \\
\text { week; } \\
\text { F: number of binge drinking } \\
\text { occasions in past month }\end{array}$ & 6,12 \\
\hline Fleming et $a^{59}$ & USA & $986,51 \%, 21$ & GP & GP & $\begin{array}{l}1 \\
\mathrm{C}\end{array}$ & $\begin{array}{l}\text { Branded } \\
\text { Information }\end{array}$ & $30(2)$ & $\begin{array}{l}\text { Q: mean number of drinks; } \\
\text { F: mean number of drinking } \\
\text { days; mean number of heavy } \\
\text { drinking days (past } 28 \text { days) }\end{array}$ & 6 \\
\hline Freyer-Adam et $a^{60}$ & Germany & $595,6 \%, 41$ & Hospital & $\begin{array}{l}\text { GP } \\
\text { Different } \\
\text { providers* }^{*}\end{array}$ & $\begin{array}{l}1 \\
2 \\
C\end{array}$ & $\begin{array}{l}\text { MI } \\
\text { MI } \\
\text { TAU }\end{array}$ & $\begin{array}{l}\text { unclear (1) } \\
78 \text { (1) }\end{array}$ & $\begin{array}{l}\text { Q: average daily alcohol intake } \\
(\mathrm{g}) \text {; total alcohol intake in past } \\
\text { week }(\mathrm{g})\end{array}$ & 12 \\
\hline
\end{tabular}

\section{Author}

BA

MI plus

Assessment

Nothing

$\begin{array}{llll}\begin{array}{l}\text { Community } \\ \text { (military) }\end{array} & \text { Counsellor } & 1 & \text { Ml } \\ & \text { C } & \text { Assessment }\end{array}$

$15.8(2)$

Branded

MI

$\mathrm{MI}$

Unclear (1) past 6 months;

change in per cent days heavy drinking

7 days

36,48

week;

Q: mean number of drinks

number of drinking

drinking days (past 28 days)

(g); total alcohol intake in past

Continued 


\begin{tabular}{|c|c|c|c|c|c|c|c|c|c|}
\hline \multirow[b]{2}{*}{ Author } & \multirow[b]{2}{*}{ Country } & \multirow{2}{*}{$\begin{array}{l}\text { Sample } \\
\mathrm{n}^{\dagger} \text { Per cent of } \mathrm{F}, \\
\text { age (years) }\end{array}$} & \multicolumn{5}{|l|}{ Intervention } & \multicolumn{2}{|l|}{ Outcomes } \\
\hline & & & Setting & Provider & Arm & Content & $\begin{array}{l}\text { Total } \\
\text { minutes } \\
\text { (sessions) }\end{array}$ & $\begin{array}{l}\text { Definition ( } Q=\text { quantity, } \\
F=\text { frequency) }\end{array}$ & $\begin{array}{l}\text { Time } \\
\text { (months) }\end{array}$ \\
\hline Gaume et $a f^{61}$ & Switzerland & $572,0 \%, 19.9$ & $\begin{array}{l}\text { Community } \\
\text { (military) }\end{array}$ & Counsellor & $\begin{array}{l}1 \\
2 \\
\mathrm{C}\end{array}$ & $\begin{array}{l}\mathrm{Ml}^{\wedge} \\
\mathrm{Ml}^{\wedge} \\
\text { Assessment }\end{array}$ & $\begin{array}{l}21.8(1) \\
21.8(1)\end{array}$ & $\begin{array}{l}\text { Q: mean change in number of } \\
\text { standard ( } 10 \mathrm{~g} \text { of alcohol) } \\
\text { drinks per week; } \\
\text { F: mean change in heavy } \\
\text { episodes ( } 6 \text { drinks or more) per } \\
\text { month }\end{array}$ & 6 \\
\hline Gaume et al $2014^{62}$ & Switzerland & $431,0 \%, 19$ & $\begin{array}{l}\text { Community } \\
\text { (military) }\end{array}$ & $\begin{array}{l}\text { Different } \\
\text { providers }\end{array}$ & $\begin{array}{l}1 \\
\mathrm{C}\end{array}$ & $\begin{array}{l}\text { MI } \\
\text { Assessment }\end{array}$ & $20-30(1)$ & $\begin{array}{l}\text { Q: number of drinks/day } \\
\text { F: number of drinking days/week }\end{array}$ & 3 \\
\hline Gentilello et $a^{63}$ & USA & $762,18 \%, 35.4$ & A\&E & Counsellor & $\begin{array}{l}1 \\
\mathrm{C}\end{array}$ & $\begin{array}{l}\text { MI } \\
\text { Assessment }\end{array}$ & $30(1)$ & $\begin{array}{l}\text { Q: changes in the number of } \\
\text { drinks consumed per week }\end{array}$ & 6,12 \\
\hline $\begin{array}{l}\text { Gottlieb-Hansen } \\
\text { et } a{ }^{64}\end{array}$ & Denmark & $772,49 \%, 60$ & $\begin{array}{l}\text { Community } \\
\text { (research) }\end{array}$ & $\begin{array}{l}\text { Different } \\
\text { providers }\end{array}$ & $\begin{array}{l}1 \\
\mathrm{C}\end{array}$ & $\begin{array}{l}\text { MI } \\
\text { Information }\end{array}$ & $15(2)$ & Q: number of drinks per week & 6,12 \\
\hline Heather et $a^{65}$ & UK & $104,25 \%, 36.4$ & GP & GP & $\begin{array}{l}1 \\
2 \\
C\end{array}$ & $\begin{array}{l}\text { Branded } \\
\text { BA } \\
\text { Assessment }\end{array}$ & NR & $\begin{array}{l}\text { Q: heaviest months } \\
\text { consumption in past } 6 \text { months } \\
\text { (units); past month's } \\
\text { consumption (units) }\end{array}$ & 6 \\
\hline Holloway et a ${ }^{66}$ & UK & $215,15 \%, 44$ & Hospital & $\begin{array}{l}\text { Nurse } \\
\text { Media } \\
\text { NA }\end{array}$ & $\begin{array}{l}1 \\
2 \\
\mathrm{C}\end{array}$ & $\begin{array}{l}\text { MI } \\
\text { Media } \\
\text { TAU }\end{array}$ & $\begin{array}{l}20(1) \\
\text { NR }\end{array}$ & $\begin{array}{l}\text { Q: change from baseline in } \\
\text { alcohol units in the past } 7 \text { days; } \\
\text { Change in maximum units in } \\
1 \text { day } \\
\text { F: change in drink days in past } \\
\text { week }\end{array}$ & 6 \\
\hline Ingersoll et $a^{{ }^{7}}$ & USA & $217,100 \%, 27.9$ & $\begin{array}{l}\text { Community } \\
\text { (research) }\end{array}$ & $\begin{array}{l}\text { Counsellor } \\
\text { NA }\end{array}$ & $\begin{array}{l}1 \\
\mathrm{C}\end{array}$ & $\begin{array}{l}\text { MI, branded } \\
\text { Information }\end{array}$ & $60(1)$ & Q: drinks per drinking day & 3,6 \\
\hline Juarez et $a \rho^{8}$ & USA & $122,53 \%, 19.4$ & College & $\begin{array}{l}\text { Counsellor } \\
\text { Counsellor } \\
\text { Media } \\
\text { Counsellor } \\
\text { NA }\end{array}$ & $\begin{array}{l}1 \\
2 \\
3 \\
4 \\
\text { C }\end{array}$ & $\begin{array}{l}\text { BA, MI } \\
\text { BA, MI } \\
\text { BA, MI } \\
\text { BA, MI } \\
\text { Assessment }\end{array}$ & $\begin{array}{l}60-80(1) \\
40-60(1) \\
\text { Unclear (1) } \\
40-60(1)\end{array}$ & Q: drinks per day & 2 \\
\hline Kulesza et a/ ${ }^{\rho 9}$ & USA & $114,72 \%, 20$ & College & Counsellor & $\begin{array}{l}1 \\
2 \\
\mathrm{C}\end{array}$ & $\begin{array}{l}\text { MI plus } \\
\text { MI plus } \\
\text { Waiting List }\end{array}$ & $\begin{array}{l}10(1) \\
50(1)\end{array}$ & Q: the DDQ & 6 weeks \\
\hline Kulesza et $a l^{70}$ & USA & $268,71 \%, 20$ & College & Counsellor & $\begin{array}{l}1 \\
2 \\
\mathrm{C}\end{array}$ & $\begin{array}{l}\text { MI plus } \\
\text { MI plus } \\
\text { Discussion }\end{array}$ & $\begin{array}{l}10(1) \\
50(1)\end{array}$ & Q: average no. drinks/week & $\begin{array}{l}4 \text { weeks } \\
(P-I)\end{array}$ \\
\hline Larimer et $a l^{71}$ & USA & $159, N R<18.8$ & College & Peer & $\begin{array}{l}1 \\
2 \\
C\end{array}$ & $\begin{array}{l}\text { MI plus } \\
\text { MI plus } \\
\text { TAU }\end{array}$ & $\begin{array}{l}60(1) \\
60(1)\end{array}$ & $\begin{array}{l}\text { Q: number of drinks over past } \\
\text { month; total average use; } \\
\text { F: frequency of use }\end{array}$ & 12 \\
\hline
\end{tabular}




\begin{tabular}{|c|c|c|c|c|c|c|c|c|c|}
\hline \multirow[b]{2}{*}{ Author } & \multirow[b]{2}{*}{ Country } & \multirow{2}{*}{$\begin{array}{l}\text { Sample } \\
\mathrm{n}^{\dagger} \text { Per cent of } \mathrm{F} \text {, } \\
\text { age (years) }\end{array}$} & \multicolumn{5}{|c|}{ Intervention } & \multicolumn{2}{|l|}{ Outcomes } \\
\hline & & & Setting & Provider & Arm & Content & $\begin{array}{l}\text { Total } \\
\text { minutes } \\
\text { (sessions) }\end{array}$ & $\begin{array}{l}\text { Definition }(Q=q u a n t i t y, \\
F=\text { frequency) }\end{array}$ & $\begin{array}{l}\text { Time } \\
\text { (months) }\end{array}$ \\
\hline Liu et $a l^{72}$ & Taiwan & $616,0 \%, 41$ & $A \& E$ & Counsellor & $\begin{array}{l}1 \\
C\end{array}$ & $\begin{array}{l}\text { MI } \\
\text { TAU }\end{array}$ & $60(2)$ & $\begin{array}{l}\text { Q: number of drinks in past } \\
3 \text { months ( } 4 \text { times a day); } \\
\text { F: number of days heavy } \\
\text { drinking ( } \geq 5 \text { drinks) in past } \\
3 \text { months ( } 4 \text { times a day); } \\
\text { number of days heavy drinking } \\
\text { in the previous week (TFLB) }\end{array}$ & 4 \\
\hline Lock et $a l^{73}$ & UK & $127,100 \%, 44.1$ & GP & Nurse & $\begin{array}{l}1 \\
\mathrm{C}\end{array}$ & $\begin{array}{l}\text { Branded } \\
\text { TAU }\end{array}$ & $5-10$ & Q: units per week & $12(P-I)$ \\
\hline Maisto et $a l^{74}$ & USA & $301,31 \%, 45.6$ & GP & $\begin{array}{l}\text { Researcher } \\
\text { Counsellor } \\
\text { NA }\end{array}$ & $\begin{array}{l}1 \\
2 \\
3\end{array}$ & $\begin{array}{l}\text { BA } \\
\text { MI } \\
\text { Control }\end{array}$ & $\begin{array}{l}10-15(1) \\
60-85(1)\end{array}$ & $\begin{array}{l}\text { Q: number of drinks in past } \\
30 \text { days } \\
\text { F: number of days of } 1-6 \text { drinks } \\
\text { in past } 30 \text { days; }\end{array}$ & 6,12 \\
\hline Murphy et $a l^{75}$ & USA & $99,54 \%, 19.6$ & College & $\begin{array}{l}\text { Counsellor } \\
\text { Counsellor } \\
\text { NA }\end{array}$ & $\begin{array}{l}1 \\
2 \\
C\end{array}$ & $\begin{array}{l}\text { MI } \\
\text { BA } \\
\text { Assessment }\end{array}$ & $\begin{array}{l}45(1) \\
50(1)\end{array}$ & $\begin{array}{l}\text { Q: drinks per week; } \\
\text { F: binge drinking days per week } \\
\text { (4+ drinks for women; } 5+\text { drinks } \\
\text { for men); drinking days per } \\
\text { week }\end{array}$ & 9 \\
\hline Noknoy et al ${ }^{76}$ & Thailand & $59,9 \%, 37$ & GP & Nurse & $\begin{array}{l}1 \\
C\end{array}$ & $\begin{array}{l}\text { MI } \\
\text { Assessment }\end{array}$ & $45(3)$ & $\begin{array}{l}\text { Q: average drinking per drinking } \\
\text { day during the previous week } \\
\text { (drinks/drinking day) }\end{array}$ & 6 \\
\hline Richmond et $\mathrm{al}^{77}$ & Australia & $378,43 \%, 37.7$ & GP & GP & $\begin{array}{l}1 \\
2 \\
C\end{array}$ & $\begin{array}{l}\text { Branded } \\
\text { BA } \\
\text { Nothing }\end{array}$ & $\begin{array}{l}30-55(1) \\
5(1)\end{array}$ & $\begin{array}{l}\text { Q: no units of ethanol in the } \\
\text { past } 7 \text { days }\end{array}$ & 6,12 \\
\hline Rubio et $a l^{88}$ & Spain & $752,35 \%, 18-65$ & GP & GP & $\begin{array}{l}1 \\
\mathrm{C}\end{array}$ & $\begin{array}{l}\text { Branded } \\
\text { Information }\end{array}$ & $20-30(2)$ & $\begin{array}{l}\text { Q: number of drinks in past } \\
7 \text { days (mean/SD); } \\
\text { F: number of binge episodes } \\
\text { (past } 30 \text { days) (mean/SD) ( }>4 \\
\text { drinks for women and } 5 \text { for men } \\
\text { in a single occasion) }\end{array}$ & 12 \\
\hline Rubio et $\mathrm{al}^{79}$ & USA & $330,100 \%, 24$ & GP & $\begin{array}{l}\text { Different } \\
\text { providers }\end{array}$ & $\begin{array}{l}1 \\
2\end{array}$ & $\begin{array}{l}\text { MI } \\
\text { Control }\end{array}$ & $70(1)$ & Q: drinks per day & $\begin{array}{l}6 \text { weeks, } \\
6,12 \mathrm{PP}\end{array}$ \\
\hline Saitz et $a \beta^{\beta 0}$ & USA & $341,29 \%, 45$ & Hospital & Counsellor & 1 & $\begin{array}{l}\text { MI } \\
\text { TAU }\end{array}$ & $30(1)$ & $\begin{array}{l}\text { Q: change decrease in number } \\
\text { drinks/day } \\
\text { F: change decrease in heavy } \\
\text { drinking episodes }\end{array}$ & 12 \\
\hline
\end{tabular}




\begin{tabular}{|c|c|c|c|c|c|c|c|c|c|}
\hline \multirow[b]{2}{*}{ Author } & \multirow[b]{2}{*}{ Country } & \multirow{2}{*}{$\begin{array}{l}\text { Sample } \\
\mathrm{n}^{\dagger} \text { Per cent of } \mathrm{F} \text {, } \\
\text { age (years) }\end{array}$} & \multicolumn{5}{|l|}{ Intervention } & \multicolumn{2}{|l|}{ Outcomes } \\
\hline & & & Setting & Provider & Arm & Content & $\begin{array}{l}\text { Total } \\
\text { minutes } \\
\text { (sessions) }\end{array}$ & $\begin{array}{l}\text { Definition }(Q=q u a n t i t y, \\
F=\text { frequency) }\end{array}$ & $\begin{array}{l}\text { Time } \\
\text { (months) }\end{array}$ \\
\hline Shiles et $a^{\beta 3}$ & UK & 154, NR, 51 & Hospital & Nurse & $\begin{array}{l}1 \\
\mathrm{C}\end{array}$ & $\begin{array}{l}\text { BA } \\
\text { TAU }\end{array}$ & $10(1)$ & $\begin{array}{l}\text { Q: daily units of alcohol in past } \\
\text { week }\end{array}$ & 3,12 \\
\hline Smith et $a^{\beta 4}$ & UK & $151,0 \%, 24$ & Hospital & Nurse & $\begin{array}{l}1 \\
\mathrm{C}\end{array}$ & $\begin{array}{l}\text { MI } \\
\text { TAU }\end{array}$ & NR & $\begin{array}{l}\text { Q: 84-day alcohol consumption; } \\
\text { alcohol consumption in a typical } \\
\text { week }\end{array}$ & 3,12 \\
\hline Walters et $a^{\beta 6}$ & USA & 279 & College & $\begin{array}{l}\text { Counsellor } \\
\text { Counsellor } \\
\text { Media }\end{array}$ & 1 & $\begin{array}{l}\text { MI (no } \\
\text { feedback) } \\
\text { Ml } \\
\text { (feedback) } \\
\text { Assessment }\end{array}$ & $40(1)$ & Q: number of drinks per week & 3,6 \\
\hline Watt et a ${ }^{\beta 7}$ & UK & 269 & $\begin{array}{l}\text { Community } \\
\text { (CJS) }\end{array}$ & $\begin{array}{l}\text { Different } \\
\text { providers }\end{array}$ & $\begin{array}{l}1 \\
\mathrm{C}\end{array}$ & $\begin{array}{l}\mathrm{Ml} \\
\mathrm{NR}\end{array}$ & $15-20$ & $\begin{array}{l}\text { Q: number of units consumed } \\
\text { per week; } \\
\text { F: number of drinking days in } \\
\text { the past } 3 \text { months }\end{array}$ & $3,12(\mathrm{PI})$ \\
\hline
\end{tabular}

Sample: $\uparrow \mathrm{n}$ denotes eligible sample randomised at baseline; $F=$ female.

Setting: CJS, Criminal Justice Service; GP, general practice; A\&E, accident and emergency.

Providers: *different providers defined as (psychologist, social worker or research nurse).

Content: ^stratified by heavy episodic and non-heavy episodic users. TAU, treatment as usual; BA, brief advice; MI, motivational interviewing.

Arm: C, control group.

Outcome: four times a day, quick drinking screen; TFLB, alcohol timeline follow-back; DDQ, Daily Drinking Questionnaire.

Outcome time=all outcomes measured in months postbaseline, unless specified: PI, postintervention; wks, weeks; PP, postpartum.

HSQ, Health Survey Questionnaire; NA, not available; NR, not reported; SEC, Standard Ethanol Content Units. 
effect sizes, interventions delivered in university contexts appeared to be most effective for frequency outcomes $(-0.21,(-0.33$ to -0.08$))$. Analysis was hampered by the small numbers of studies in several categories (table 5).

When limiting the analysis to the subset of either quantity or frequency outcomes the setting of intervention did not explain heterogeneity (all joint tests of moderators $\mathrm{p}>0.10$ ).

\section{Provider}

In the model including all quantity outcomes, the provider of intervention did not meaningfully explain

\begin{tabular}{|c|c|c|}
\hline Trials & & \\
\hline \multicolumn{3}{|l|}{ Setting of intervention } \\
\hline$A \& E$ & $20 \%$ & 10 \\
\hline Non-health settings & $12 \%$ & 6 \\
\hline Ambulatory or primary care & $38 \%$ & 19 \\
\hline Hospital inpatient services & $10 \%$ & 5 \\
\hline University & $20 \%$ & 10 \\
\hline \multicolumn{3}{|l|}{ Provider } \\
\hline Counsellor/mental health clinician & $44 \%$ & 22 \\
\hline Different providers & $8 \%$ & 4 \\
\hline GP & $22 \%$ & 11 \\
\hline Nurse & $18 \%$ & 9 \\
\hline Peer intervention & $4 \%$ & 2 \\
\hline Combination & $12 \%$ & 6 \\
\hline GP and nurse & $8 \%$ & 4 \\
\hline GP and counsellor & $4 \%$ & 2 \\
\hline \multicolumn{3}{|l|}{ Content } \\
\hline Brief advice & $24 \%$ & 12 \\
\hline Motivational interviewing & $48 \%$ & 24 \\
\hline Motivational interviewing 'plus' & $40 \%$ & 20 \\
\hline \multicolumn{3}{|l|}{ Outcomes } \\
\hline Quantity & & 50 \\
\hline Mean follow-up in months (SD) & $9.0(8.3)$ & \\
\hline Quantity per unit time & $94 \%$ & 47 \\
\hline Quantity per drinking occasion & $30 \%$ & 15 \\
\hline Frequency & & 26 \\
\hline Mean follow-up in months (SD) & $\begin{array}{l}11.1 \\
(10.5)\end{array}$ & \\
\hline $\begin{array}{l}\text { Frequency of any drinking occasion per } \\
\text { unit time }\end{array}$ & $32 \%$ & 16 \\
\hline $\begin{array}{l}\text { Frequency of binge drinking occasions } \\
\text { per unit time }\end{array}$ & $30 \%$ & 15 \\
\hline
\end{tabular}

heterogeneity, based on $\mathrm{I}^{2}$ for this model (34\%). Interventions delivered at least in part by nurses appeared to have the largest effect by magnitude $(\mathrm{d}=$ $-0.23,95 \%$ CI $(-0.33$ to -0.13$)$ ), though this difference was not supported by a significant joint test of moderators (Wald $\mathrm{p}=0.09$ ).

Analyses with more specific sets of outcomes revealed a similar picture. Examination of effects at the first time point for the amount of alcohol per unit time showed that interventions delivered at least in part by the nurses $(\mathrm{d}=-0.30,95 \%$ CI $(-0.47$ to -0.12$))$ were the most effective, with a significant joint test of moderators (Wald $\mathrm{p}=0.048$; see online web appendix table 2). Interventions delivered by a range of different providers were least effective and did not yield a statistically significant effect. However, few studies were included in this category of providers. The provider of intervention explained some heterogeneity when the analysis was limited to the amount of alcohol per unit time (residual $\mathrm{I}^{2}=32 \%$, Wald $\mathrm{p}=0.01$ ) but not per drinking occasion.

For frequency outcomes, the provider of intervention did not explain heterogeneity either combined (Wald $\mathrm{p}=0.17$ ) or for drinking occasion per unit time (Wald $\mathrm{p}=0.73$ ) but the effect was marginal, but non-significant, for bingeing occasions (Wald $\mathrm{p}=0.07$ ).

\section{Content}

For quantity outcomes, the content of intervention did not explain a statistically significant amount of heterogeneity (residual $\mathrm{I}^{2}=39 \%$, Wald $\mathrm{p}=0.54$ ), with little apparent reduction in $\mathrm{I}^{2}$. While all content groups had statistically significant mean effects, brief advice appeared to be most effective ( $d=-0.20,95 \%$ CI $(-0.30$ to -0.09$)$ ) with the impact of motivational interviewing $(\mathrm{d}=-0.13)$ and MI plus $(\mathrm{d}=-0.16)$ also statistically significant.

For frequency outcomes, the content of intervention did not explain a significant amount of heterogeneity (residual $\mathrm{I}^{2}=29 \%$, Wald $\mathrm{p}=0.48$ ). Effects by the content group for motivational interviewing were similar to those in the analysis of quantity outcomes, though brief advice did not have a statistically significant effect on the frequency of alcohol use $(-0.08(-0.26$ to 0.09$))$.

Estimates of heterogeneity remained the same when limiting the analysis to the subset of either quantity or frequency outcomes.

Table 3 Summary risk of bias assessment

\begin{tabular}{lccc}
\hline & \multicolumn{3}{l}{ Score-proportion (number of estimates) } \\
\cline { 2 - 4 } Risk of bias indicator & High risk \% (k) & Low risk \% (k) & Unclear risk \% (k) \\
\hline Allocation concealment & $2(1)$ & $72(36)$ & $26(13)$ \\
Blinding of participants and providers & $12(6)$ & $30(15)$ & $58(29)$ \\
Blinding of outcome assessment & $10(5)$ & $42(21)$ & $48(24)$ \\
Intention-to-treat analysis & $6(3)$ & $48(24)$ & $46(23)$ \\
Loss to follow-up & $20(10)$ & $80(40)$ & $0(0)$ \\
\hline
\end{tabular}


Table 4 Results of multilevel metaregression for quantity outcomes

\begin{tabular}{|c|c|c|c|c|c|c|c|c|c|c|c|c|c|}
\hline \multirow[b]{2}{*}{ Outcomes } & \multirow[b]{2}{*}{ Group name } & \multicolumn{4}{|l|}{ All quantity outcomes } & \multicolumn{4}{|c|}{ Quantity of alcohol per unit time } & \multicolumn{4}{|c|}{ Quantity of alcohol per drinking occasion } \\
\hline & & ES (95\% Cl) & $k(n)$ & $I^{2}(\%)$ & p Value & $\overline{\mathrm{ES}}(95 \% \mathrm{Cl})$ & $k(n)$ & $1^{2}(\%)$ & p Value & ES (95\% Cl) & $k(n)$ & $I^{2}(\%)$ & p Value \\
\hline Overall & Mean effect & $-0.15(-0.20$ to -0.10$)$ & $50(268)$ & 37 & & $-0.17(-0.22$ to -0.12$)$ & $47(144)$ & 38 & & $-0.10(-0.18$ to -0.01$)$ & $15(59)$ & 36 & \\
\hline \multirow{2}{*}{$\begin{array}{l}\text { Overall, } \\
\text { time-adjusted }\end{array}$} & Mean effect & $-0.15(-0.20$ to -0.11$)$ & $50(268)$ & 36 & 0.03 & $-0.17(-0.22$ to -0.12$)$ & $47(144)$ & 38 & 0.21 & $-0.11(-0.19$ to -0.03$)$ & $15(59)$ & 34 & 0.09 \\
\hline & Time (month) & $0.003(0.0003$ to 0.006$)$ & & & & $0.002(-0.001$ to 0.006$)$ & & & & $0.005(-0.001$ to 0.01$)$ & & & \\
\hline \multirow{5}{*}{$\begin{array}{l}\text { Setting of } \\
\text { intervention }\end{array}$} & $A \& E$ & $-0.10(-0.19$ to -0.002$)$ & $10(44)$ & 34 & 0.12 & $-0.12(-0.22$ to -0.01$)$ & $9(26)$ & 37 & 0.17 & $-0.001(-0.14$ to 0.13$)$ & $4(8)$ & 28 & 0.17 \\
\hline & $\begin{array}{l}\text { Ambulatory } \\
\text { or primary } \\
\text { care }\end{array}$ & $-0.20(-0.27$ to -0.13$)$ & $19(84)$ & & & $-0.22(-0.29$ to -0.14$)$ & $19(51)$ & & & $-0.14(-0.25$ to -0.03$)$ & $7(18)$ & & \\
\hline & $\begin{array}{l}\text { Hospital } \\
\text { inpatient } \\
\text { services }\end{array}$ & $-0.14(-0.29$ to 0.01$)$ & $5(13)$ & & & $-0.15(-0.31$ to 0.006$)$ & $5(12)$ & & & NA & NA & & \\
\hline & $\begin{array}{l}\text { Non-health } \\
\text { settings }\end{array}$ & $-0.03(-0.16$ to 0.10$)$ & $6(15)$ & & & $-0.04(-0.18$ to 0.11$)$ & $5(11)$ & & & $-0.01(-0.30$ to 0.29$)$ & $1(4)$ & & \\
\hline & University & $-0.20(-0.39$ to -0.09$)$ & $10(112)$ & & & $-0.21(-0.23$ to -0.09$)$ & $9(44)$ & & & $-0.22(-0.39$ to -0.06$)$ & $3(29)$ & & \\
\hline \multirow[t]{5}{*}{ Provider } & $\begin{array}{l}\text { Counsellor/ } \\
\text { mental health } \\
\text { clinician }\end{array}$ & $-0.11(-0.17$ to -0.05$)$ & $24(163)$ & 34 & 0.09 & $-0.10(-0.17$ to -0.04$)$ & $22(79)$ & 32 & 0.01 & $-0.11(-0.23$ to 0.01$)$ & $8(41)$ & 43 & 0.67 \\
\hline & $\begin{array}{l}\text { Different } \\
\text { providers }\end{array}$ & $-0.12(-0.27$ to 0.03$)$ & $4(10)$ & & & $-0.12(-0.25$ to 0.02$)$ & $4(10)$ & & & NA & & & \\
\hline & Physician & $-0.12(-0.20$ to -0.04$)$ & $17(65)$ & & & $-0.14(-0.22$ to -0.06$)$ & $17(40)$ & & & $0.02(-0.16$ to 0.21$)$ & $6(10)$ & & \\
\hline & Nurse & $-0.23(-0.33$ to -0.13$)$ & $13(41)$ & & & $-0.28(-0.38$ to -0.18$)$ & $12(29)$ & & & $-0.18(-0.37$ to -0.003$)$ & $5(9)$ & & \\
\hline & $\begin{array}{l}\text { Peer } \\
\text { intervention }\end{array}$ & $-0.08(-0.29$ to 0.13$)$ & $2(10)$ & & & $-0.05(-0.28$ to 0.17$)$ & $2(3)$ & & & $-0.004(-0.28$ to 0.27$)$ & $2(3)$ & & \\
\hline \multirow[t]{3}{*}{ Content } & Brief advice & $-0.20(-0.31$ to -0.09$)$ & $12(26)$ & 39 & 0.54 & $-0.22(-0.34$ to -0.11$)$ & $11(18)$ & 59 & 0.31 & $-0.16(-0.37$ to 0.05$)$ & $3(6)$ & 43 & 0.89 \\
\hline & $\begin{array}{l}\text { Motivational } \\
\text { interviewing }\end{array}$ & $-0.13(-0.19$ to -0.07$)$ & $24(132)$ & & & $-0.13(-0.20$ to -0.07$)$ & $24(73)$ & & & $-0.11(-0.22$ to 0.004$)$ & $9(28)$ & & \\
\hline & $\begin{array}{l}\text { Motivational } \\
\text { interviewing } \\
\text { plus }\end{array}$ & $-0.16(-0.23$ to -0.09$)$ & $20(110)$ & & & $-0.19(-0.27$ to -0.11$)$ & $17(53)$ & & & $-0.10(-0.24$ to 0.03$)$ & $6(25)$ & & \\
\hline
\end{tabular}

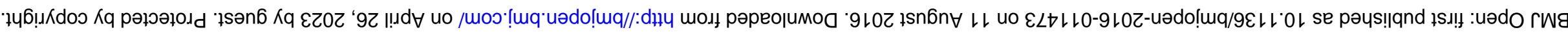


Table 5 Results of multilevel metaregression for frequency outcomes

\begin{tabular}{|c|c|c|c|c|c|c|c|c|c|c|c|c|c|}
\hline \multirow[b]{2}{*}{ Outcomes } & \multirow[b]{2}{*}{ Group name } & \multicolumn{4}{|l|}{ All quantity outcomes } & \multicolumn{4}{|c|}{ Quantity of alcohol per unit time } & \multicolumn{4}{|c|}{ Quantity of alcohol per drinking occasion } \\
\hline & & ES $(95 \%$ Cl) & $k(n)$ & $I^{2}(\%)$ & p Value & ES $(95 \% \mathrm{Cl})$ & $k(n)$ & $I^{2}(\%)$ & $p$ Value & ES (95\% Cl) & $k(n)$ & $I^{2}(\%)$ & p Value \\
\hline Overall & Mean effect & $-0.15(-0.20$ to -0.11$)$ & $26(114)$ & 23 & & $-0.12(-0.19$ to -0.06$)$ & $16(38)$ & 23 & & $-0.17(-0.23$ to -0.11$)$ & $15(76)$ & 20 & \\
\hline \multirow{2}{*}{$\begin{array}{l}\text { Overall, } \\
\text { time-adjusted }\end{array}$} & Mean effect & $-0.16(-0.20$ to -0.11$)$ & $26(114)$ & 23 & 0.36 & $-0.12(-0.19$ to -0.06$)$ & $16(38)$ & 24 & 0.55 & $-0.18(-0.24$ to -0.11$)$ & $15(76)$ & 20 & 0.56 \\
\hline & Time (month) & $0.002(-0.002$ to 0.005$)$ & & & & $0.002(-0.004$ to 0.007$)$ & & & & $0.001(-0.003$ to 0.006$)$ & & & \\
\hline \multirow{5}{*}{$\begin{array}{l}\text { Setting of } \\
\text { intervention }\end{array}$} & $A \& E$ & $-0.11(-0.21$ to -0.005$)$ & $5(26)$ & 25 & 0.54 & $-0.13(-0.26$ to -0.0002$)$ & $4(12)$ & 28 & 0.41 & $-0.11(-0.22$ to 0.01$)$ & $3(14)$ & 20 & 0.25 \\
\hline & $\begin{array}{l}\text { Ambulatory } \\
\text { or primary } \\
\text { care }\end{array}$ & $-0.18(-0.26$ to -0.10$)$ & $10(40)$ & & & $-0.07(-0.19$ to 0.06$)$ & $5(12)$ & & & $-0.24(-0.33$ to -0.15$)$ & $6(28)$ & & \\
\hline & $\begin{array}{l}\text { Hospital } \\
\text { inpatient } \\
\text { services }\end{array}$ & $-0.21(-0.47$ to 0.04$)$ & $2(2)$ & & & $-0.50(-0.94$ to -0.06$)$ & $1(1)$ & & & $-0.07(-0.37$ to 0.23$)$ & $1(1)$ & & \\
\hline & $\begin{array}{l}\text { Non-health } \\
\text { settings }\end{array}$ & $-0.08(-0.22$ to 0.06$)$ & $4(7)$ & & & $-0.11(-0.32$ to 0.11$)$ & $2(3)$ & & & $-0.06(-0.24$ to 0.13$)$ & $2(4)$ & & \\
\hline & University & $-0.21(-0.33$ to -0.08$)$ & $5(39)$ & & & $-0.18(-0.36$ to -0.003$)$ & $4(10)$ & & & $-0.21(-0.37$ to -0.05$)$ & $3(29)$ & & \\
\hline \multirow[t]{5}{*}{ Provider } & $\begin{array}{l}\text { Counsellor/ } \\
\text { mental health } \\
\text { clinician }\end{array}$ & $-0.11(-0.17$ to -0.04$)$ & $14(73)$ & 23 & 0.17 & $-0.12(-0.22$ to -0.02$)$ & $9(25)$ & 32 & 0.73 & $-0.12(-0.20$ to -0.05$)$ & $9(48)$ & 18 & 0.07 \\
\hline & $\begin{array}{l}\text { Different } \\
\text { providers }\end{array}$ & $-0.24(-0.52$ to 0.03$)$ & $1(1)$ & & & $-0.25(-0.56$ to 0.07$)$ & $1(1)$ & & & NA & & & \\
\hline & Physician & $-0.13(-0.22$ to -0.04$)$ & $10(30)$ & & & $-0.03(-0.19$ to 0.13$)$ & $6(8)$ & & & $-0.18(-0.28$ to -0.07$)$ & $5(22)$ & & \\
\hline & Nurse & $-0.19(-0.31$ to -0.07$)$ & 7 (22) & & & $-0.20(-0.31$ to 0.01$)$ & $4(5)$ & & & $-0.17(-0.31$ to -0.02$)$ & $3(17)$ & & \\
\hline & $\begin{array}{l}\text { Peer } \\
\text { intervention }\end{array}$ & $-0.06(-0.27$ to 0.13$)$ & $2(3)$ & & & $-0.08(-0.31$ to 0.16$)$ & 2 (3) & & & NA & & & \\
\hline \multirow[t]{3}{*}{ Content } & Brief advice & $-0.08(-0.26$ to 0.09$)$ & $3(7)$ & 29 & 0.48 & $0.17(-0.11$ to 0.44$)$ & $2(4)$ & 26 & 0.10 & $-0.23(-0.44$ to -0.02$)$ & $2(3)$ & 26 & 0.52 \\
\hline & $\begin{array}{l}\text { Motivational } \\
\text { interviewing }\end{array}$ & $-0.15(-0.21$ to -0.08$)$ & $15(58)$ & & & $-0.15(-0.23$ to -0.06$)$ & $9(20)$ & & & & $9(38)$ & & \\
\hline & $\begin{array}{l}\text { Motivational } \\
\text { interviewing } \\
\text { plus }\end{array}$ & $-0.19(-0.27$ to -0.11$)$ & $11(49)$ & & & $-0.13(-0.24$ to -0.03$)$ & $7(14)$ & & & $-0.21(-0.31$ to -0.11$)$ & $6(35)$ & & \\
\hline
\end{tabular}

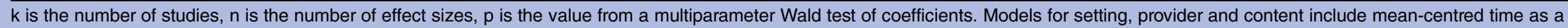
covariate, but not in the multiparameter Wald test.

A\&E, accident and emergency; ES, effect size; NA, not available. 
Sensitivity check: metaregression on subset of outcomes by first and last time point

Overall effect estimates based on first and last time point were similar to the corresponding value reported in the main analysis, but estimates of heterogeneity (measured through $\mathrm{I}^{2}$ ) tend to be higher. Setting of intervention explained some heterogeneity for the alcohol per unit time outcome at the first time of marginal significance (residual $\mathrm{I}^{2}=49 \%$, Wald $\mathrm{p}=0.08$ ). Findings also suggest that the provider explained some heterogeneity (residual $\mathrm{I}^{2}=43 \%$, Wald $\mathrm{p}=0.05$ ) with nurses having the biggest effect $(\mathrm{d}=-30,95 \%$ CI $(-0.41$ to -0.20$))$ and interventions delivered by different providers had the least effect $(\mathrm{d}=-0.07,95 \%$ CI $(-0.12$ to -0.03$))$. The content of intervention explained some heterogeneity (residual $\mathrm{I}^{2}=43 \%$, Wald $\mathrm{p}=0.04$ ), brief advice was the most effective $(\mathrm{d}=-0.25,95 \%$ CI $(-0.42$ to -0.07$))$ and motivational interviewing was least effective $(\mathrm{d}=-0.09$, $95 \%$ CI $(-0.15$ to -0.04$)$; figures $3-5)$. With the exception of content, evidence of heterogeneity did not remain significant at the last time point. There was no evidence of heterogeneity for alcohol consumed per drinking occasion or for either subset of frequency outcomes. All findings are summarised in the online web appendix tables 2-5.

\section{DISCUSSION}

Our findings provide important new evidence on how the effectiveness of brief alcohol interventions differs by setting, provider and content, informing us of optimum modality. Our findings show that the provider of

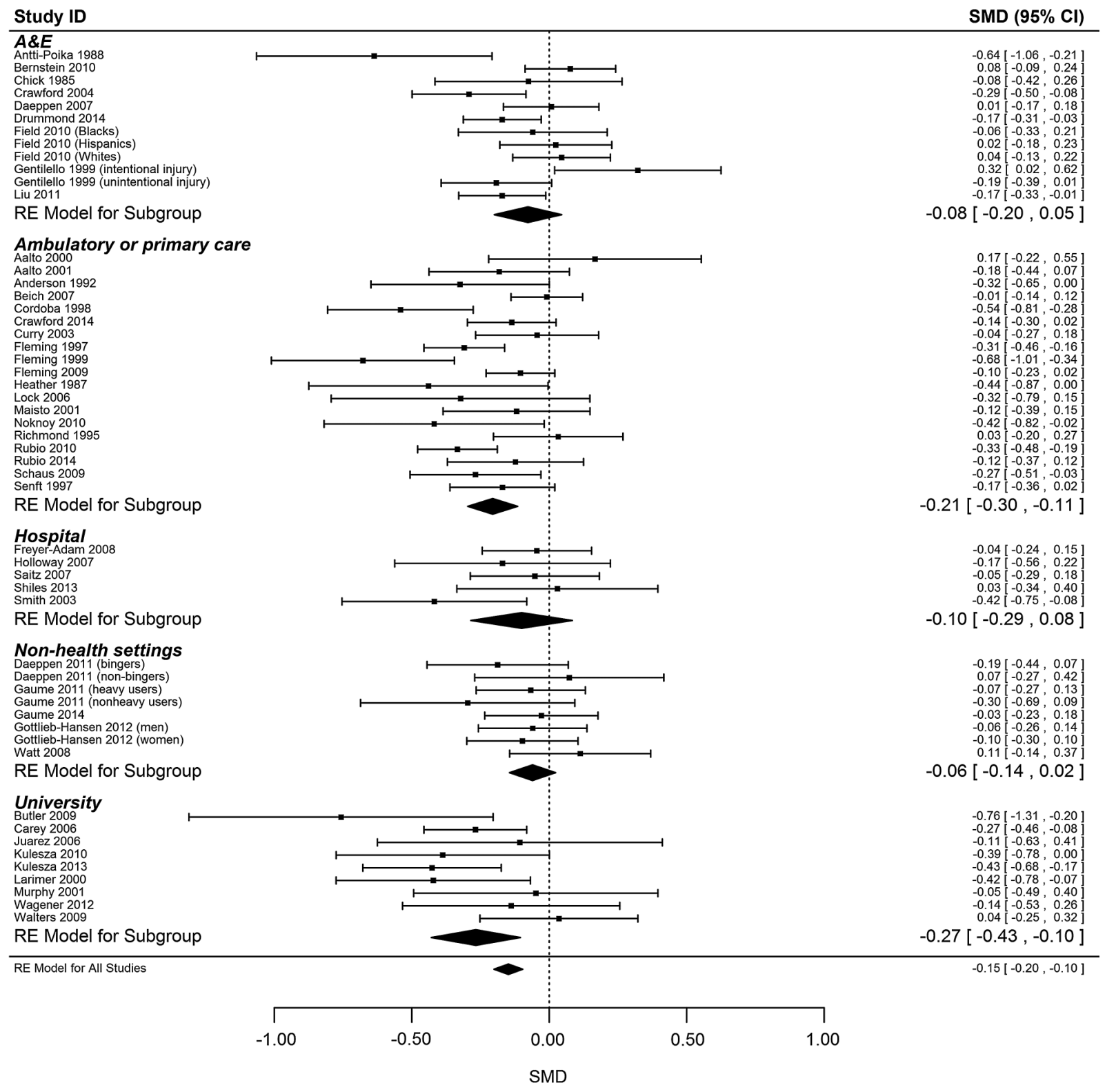

Figure 3 Metaregression analysis on alcohol consumed per unit time at first follow-up by setting of intervention. A\&E, accident and emergency. 


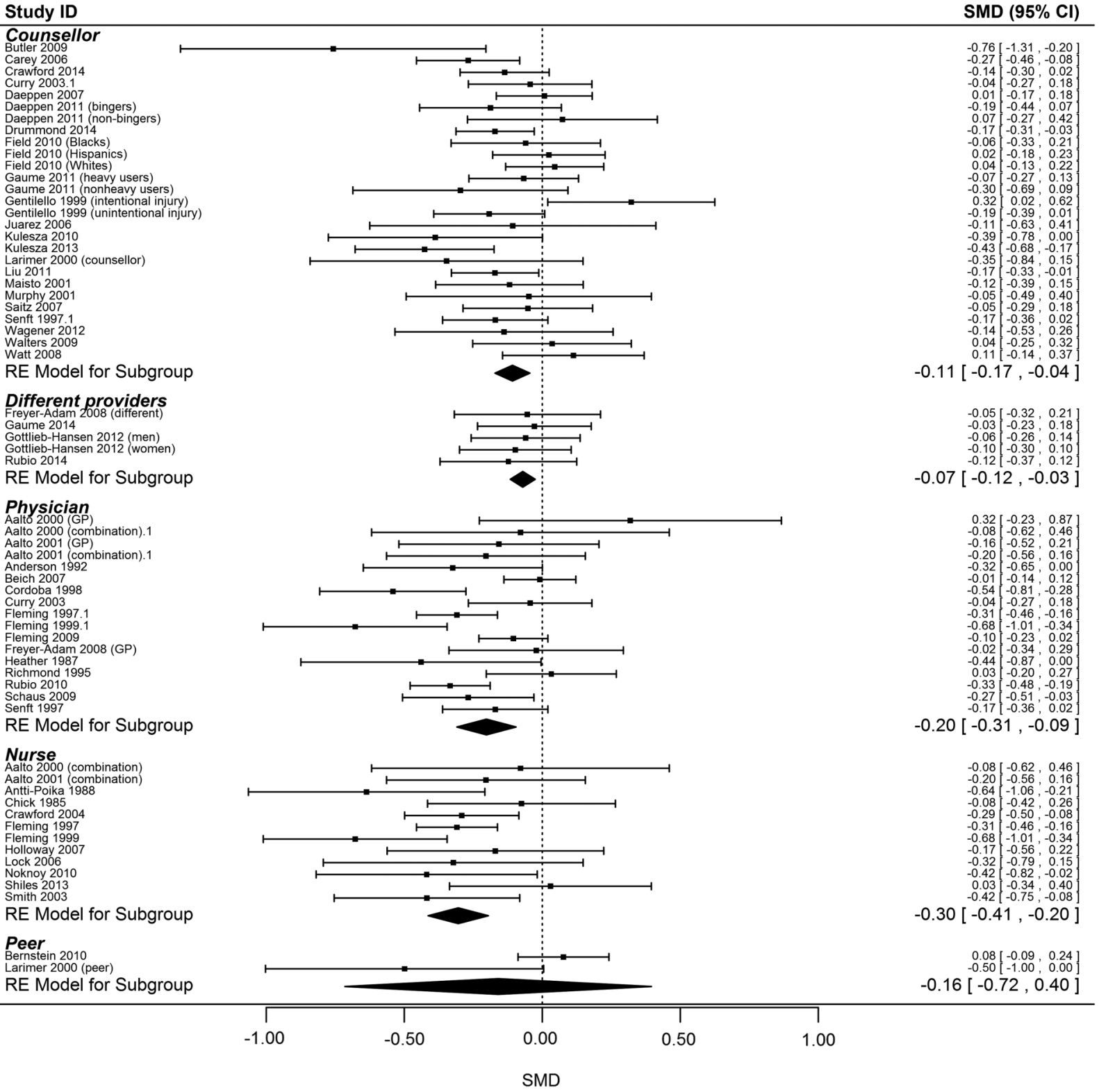

Figure 4 Metaregression analysis on alcohol consumed per unit time at first follow-up by provider of intervention.

intervention may matter. We observed some reductions in heterogeneity in the multilevel analysis of amount of alcohol consumed per unit time, and interventions delivered by nurses having the most effect in reducing the quantity of alcohol consumed, but not the frequency of consumption. This finding builds on other evidence showing a modest effect of brief interventions delivered by non-physicians (nurses and healthcare workers) in primary care settings. ${ }^{24}$ We found that neither setting nor the content appeared to significantly moderate intervention effectiveness: we found little evidence on the effectiveness of brief interventions in community settings or A\&E ; brief advice was the most effective content in reducing the quantity of alcohol consumed but not the frequency of drinking and there seemed to be little difference in the effect of
MI or MI plus on either the quantity or frequency outcomes.

While the setting did not explain heterogeneity, findings show that university and ambulatory/primary care settings were the most effective in terms of magnitude of effect size, which is supported by previous reviews in this field. ${ }^{1415}{ }^{17}$ Prior research has suggested that while ABIs delivered in A\&E settings may be effective in reducing alcohol consumption among hazardous and harmful drinkers, ${ }^{19}$ it may not provide the most appropriate context for discussion on alcohol use. ${ }^{88}$ The brevity of visits, lack of privacy for the delivery of the intervention and severity of injury may hinder the interaction between the patient and the practitioner reducing effectiveness. ${ }^{88-91}$ Other evidence shows that discussion of drinking behaviours is facilitated by a good 


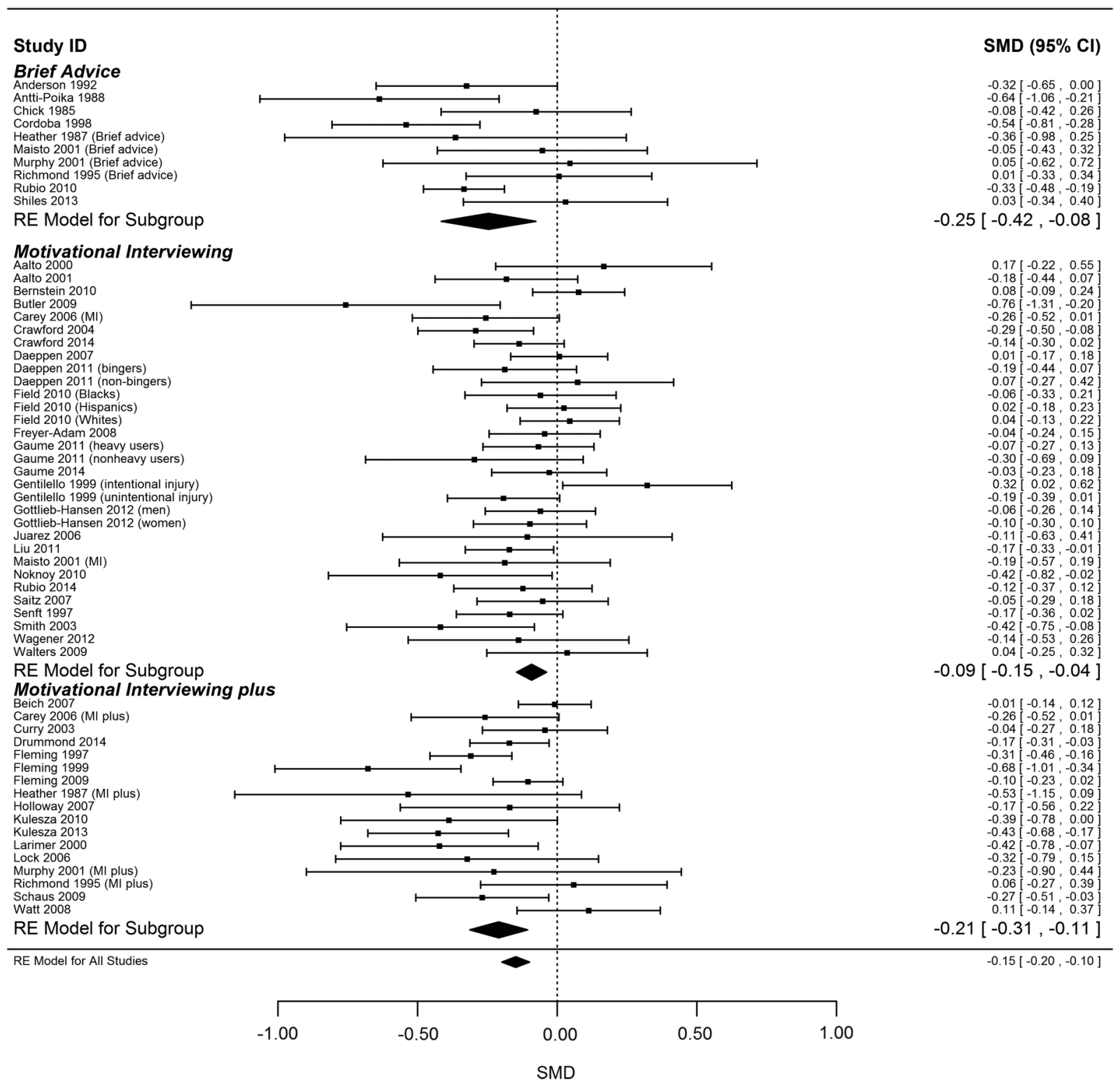

Figures 5 Metaregression analysis on alcohol consumed per unit time at first follow-up by content of intervention.

relationship between the practitioner and the client. ${ }^{73}$ Our finding of increased reduction in alcohol consumption when the intervention is delivered by a nurse is important. The majority of previous research has focused on physician-led interventions, but there is growing evidence to support the effectiveness of nurse-led interventions in primary care and other settings. ${ }^{24} 9293$ As the largest group of healthcare workers with repeated patient contact and with a health promotion remit as part of their role, they are well placed to deliver ABIs. ${ }^{92}{ }^{94}$ Barriers to nurses delivering the interventions include lack of time, worry about losing trust of the patient and inadequate training. ${ }^{95}{ }^{96}$ Resources and training should be provided to support nurses to undertake this role and embed it within services. The provision of ABIs under the category of different providers was not associated with a reduction in the consumption in alcohol. This may be related to problems with training of different providers, but the category was small and included a diverse range of providers, making the finding difficult to interpret. Similarly only a moderate effect was associated with counsellors, but again this definition encompasses a diverse group of practitioners ranging from clinical psychology students ${ }^{75}$ to alcohol workers with specialist training in alcohol counselling. ${ }^{56}$

While our categories of intervention content did not meaningfully or statistically explain heterogeneity in either quantity or frequency outcomes in the multilevel analysis, they did in the stratified analysis for first and last assessment time points. Effect sizes for quantity outcomes for all three classes of content were statistically significant, with brief advice yielding the largest effect. 
This provides important empirical evidence that brief advice can reduce alcohol intake, where evidence was lacking, and corroborates previous research that demonstrated no difference in effect between brief advice and longer motivational interviewing in reducing the harmful levels of drinking in A\&E, primary care and criminal justice settings. ${ }^{12} 569798$

\section{Strengths and limitations}

A key strength of this review is the use of a multilevel meta-analysis method to integrate all the relevant effect sizes from the included studies. This circumvented problems in other systematic reviews around the selection of specific effect sizes for meta-analysis. However, we were unable to explicitly model correlation between outcomes within studies, though simulation evidence suggests that this may not have a large impact on the estimation of intervention effects. ${ }^{99}$ We used Cohen's d to standardise outcomes. While this is common across many systematic reviews addressing continuous outcomes, it is uncommon for systematic reviews of alcohol outcomes, where standardisation is often in terms of standard drinks or grams of ethanol consumed. This may somewhat limit comparability between reviews, but it was a critical step in employing the multilevel meta-analysis model we used. As a second sensitivity analysis, we compared the findings from the multilevel model with a stratified analysis focusing on a subset of outcome variables. Findings from the two analyses were comparable. The stratified analysis of quantity of alcohol consumed per unit time suggested stronger effects of setting, provider and content of intervention at the first time point of assessment than indicated in the multilevel models but with comparable effect estimates within each category. Tests for publication bias do not yet exist for multilevel meta-analyses. While our tests using all available effect sizes did not reveal significant publication bias on either the quantity or frequency outcomes, it is unlikely that this is the best way to test publication bias in the context of dependent effect sizes. While we used the broadest categories appropriate for setting and provider of interventions, the number of studies included in the meta-analysis examining frequency outcomes meant that metaregressions were likely underpowered. We did not examine the effect of sex, ethnicity or age as a covariate since the sample size would have been too small to conduct a multivariate metaregression analysis. As the number of trials grows, this meta-analysis should be repeated in order to better estimate the differences between categories and examine the effect of other factors.

These findings should also be viewed in the context of study-level heterogeneity. In our multilevel metaanalyses, heterogeneity was surprisingly low considering the diversity of settings, providers and modalities included in this body of evidence. One possible reason for this is that because we included all relevant outcomes, we avoided some of the 'random errors' that may arise when only selecting one outcome per study. That is, including more information from each study will provide an estimate of statistical heterogeneity that more meaningfully accounts for study-level differences. This is not to say that it was inappropriate to explore this heterogeneity through structured and prehypothesised subgroup analyses, as was done here. Rather, the magnitude of difference in effects between studies may not be as pronounced as would be expected in a systematic review with such diverse interventions. While there was a low risk of bias in relation to some aspects of the study design (randomisation, loss to follow-up), there was a high percentage of unclear risk for many criteria, limiting our ability to fully assess the risk of bias. Because of the substantial number of categories for many of our metaregressions, we were unable to conduct a sensitivity analysis on risk of bias as that would have resulted in underpowered models.

Further research is needed to examine the effectiveness of ABIs in community settings. Our review suggested limited effect but relied on a small number of studies across a wide variety of settings. Our review excluded the use of computer-based interventions, which may be an important approach to reaching populations who do not consider themselves at risk. Some evidence shows that computer-delivered interventions with personalised feedback can effectively reduce alcohol consumption at short-term and long-term follow-up; however, the evidence is weaker when comparing direct feedback between face-to-face and computerised feedback. ${ }^{85}$ Our findings clearly show the importance of provider in effective delivery of $\mathrm{ABIs}$ and it will be important for future research to measure effectiveness of computerised feedback against different providers. Subsequent trials should also comprehensively describe intervention components to enable finer-grained analysis of the relationship between specific aspects of intervention modalities and their effectiveness.

Findings of this review contribute significantly to the understanding of the key processes involved in the delivery of effective ABIs, and have important policy implications for the design of preventative alcohol strategies both in the UK and internationally. The review provides important new evidence on the effectiveness of brief advice in reducing quantity of alcohol consumed and the role that nurses play in moderating the effectiveness of interventions. Resources should be prioritised to provide further support and training for nurses to deliver ABIs, as well as to undertake research to understand why nurse-led interventions are more effective so that appropriate training can be provided to other practitioners.

\section{Author affiliations}

${ }^{1}$ Department of Social and Environmental Health, Faculty of Public Health and Policy, London School of Hygiene and Tropical Medicine, London, UK

${ }^{2}$ Division of Health Sciences, Warwick Medical School, University of Warwick, Coventry, UK

${ }^{3}$ Institute of Health and Society, Newcastle University, Newcastle, UK 
${ }^{4}$ Health and Social Care Institute, Teesside University, Middlesbrough, UK ${ }^{5}$ Camden \& Islington Public Health, London Boroughs of Islington and Camden, London, UK

\section{Twitter Follow Dorothy Newbury-Birch at @dotbirch}

Acknowledgements The authors would like to acknowledge Antonio Gasparinni and Colin Muirhead who provided statistical advice on the multilevel meta-analysis and sensitivity check applied in the review.

Contributors LP and CA developed the study protocol with advice from EK. LP conducted the search with assistance from DN-B. LP and DN-B checked the eligibility criteria of all manuscripts with help from $A 0$. $A 0$ and JB conducted the data extraction and validation of extraction. GJM-T developed the statistical approach and conducted all statistical analyses in collaboration with LP. All authors commented on the manuscript.

Funding The study was funded by Camden and Islington Public Health, who commented on the study protocol, analysis and interpretation of findings.

Competing interests None declared.

Provenance and peer review Not commissioned; externally peer reviewed.

Data sharing statement No additional data are available.

Open Access This is an Open Access article distributed in accordance with the Creative Commons Attribution Non Commercial (CC BY-NC 4.0) license, which permits others to distribute, remix, adapt, build upon this work noncommercially, and license their derivative works on different terms, provided the original work is properly cited and the use is non-commercial. See: http:// creativecommons.org/licenses/by-nc/4.0/

\section{REFERENCES}

1. World Health Organisation. Global status report on alcohol and health 2014. Luxembourg: World Health Organisation, 2014.

2. GBD 2013 Mortality and Causes of Death Collaborators. Global, regional, and national age-sex specific all-cause and cause-specific mortality for 240 causes of death, 1990-2013: a systematic analysis for the Global Burden of Disease Study 2013. Lancet 2015;385:117-71.

3. Office of National Statistics. Alcohol-related deaths in the United Kingdom, registered in 2012. UK: Office of National Statistics, 2014.

4. National Audit Office. Reducing alcohol harm: health services in England for alcohol misuse. London, UK: The Stationery Office; Department of Health, 2008.

5. Heather N. Breaking new ground in the study and practice of alcoho brief interventions. Drug Alcohol Rev 2010;29:584-8.

6. Kaner EF, Beyer F, Dickinson HO, et al. Effectiveness of brief alcohol interventions in primary care populations. Cochrane Database Syst Rev 2007;(2):CD004148

7. McCambridge J, Kypri K. Can simply answering research questions change behaviour? Systematic review and meta analyses of brief alcohol intervention trials. PLOS ONE 2011;6:e23748.

8. McCambridge J, Rollnick S. Should brief interventions in primary care address alcohol problems more strongly? Addiction 2014:109:1054-8.

9. Alvarez-Bueno C, Rodriguez-Martin B, Garcia-Ortiz L, et al. Effectiveness of brief interventions in primary health care settings to decrease alcohol consumption by adult non-dependent drinkers: a systematic review of systematic reviews. Prev Med 2015;76(Suppl): S33-8.

10. Poikolainen K. Effectiveness of brief interventions to reduce alcohol intake in primary health care populations: a meta-analysis. Prev Med 1999;28:503-9.

11. Rubak SLM, Sandbaek A, Lauritzen T, et al. Motivational Interviewing: a systematic review and meta-analysis. Br J Gen Pract 2005;55:305-12.

12. Gaume J, McCambridge J, Bertholet N, et al. Mechanisms of action of brief alcohol interventions remain largely unknown-a narrative review. Front Psychiatry 2014;5:108.

13. Ballesteros J, Gonzalez-Pinto A, Querejeta I, et al. Brief interventions for hazardous drinkers delivered in primary care are equally effective in men and women. Addiction 2004;99:103-8.

14. Kaner EFS, Dickinson HO, Beyer F, et al. The effectiveness of brief alcohol interventions in primary care settings: a systematic review. Drug Alcohol Rev 2009;28:301-23.
15. O'Donnell A, Anderson P, Newbury-Birch D, et al. The impact of brief alcohol interventions in primary healthcare: a systematic review of reviews. Alcohol Alcohol 2014;49:66-78.

16. Marlatt GA, Baer JS, Kivlahan DR, et al. Screening and brief intervention for high-risk college student drinkers: results from a 2-year follow-up assessment. J Consult Clin Psychol 1998;66:604-15.

17. Carey KB, Scott-Sheldon LAJ, Carey MP, et al. Individual-level interventions to reduce college student drinking: a meta-analytic review. Addict Behav 2007;32:2469-94.

18. O'Connor MJ, Whaley SE. Brief intervention for alcohol use by pregnant women. Am J Public Health 2007;97:252-8.

19. Elzerbi C, Donoghue K, Drummond C. A comparison of the efficacy of brief interventions to reduce hazardous and harmful alcohol consumption between European and non-European countries: a systematic review and meta-analysis of randomized controlled trials. Addiction 2015;110:1082-91.

20. Havard A, Shakeshaft A, Sanson-Fisher R. Systematic review and meta-analyses of strategies targeting alcohol problems in emergency departments: interventions reduce alcohol-related injuries. Addiction 2008;103:368-76; discussion 77-8.

21. Emmen MJ, Schippers GM, Bleijenberg G, et al. Effectiveness of opportunistic brief interventions for problem drinking in a general hospital setting: systematic review. BMJ 2004;328:318.

22. McQueen J, Howe TE, Allan L, et al. Brief interventions for heavy alcohol users admitted to general hospital wards. Cochrane Database Syst Rev 2011;(8):Cd005191.

23. Nilsen $P$. Brief alcohol intervention-where to from here? Challenges remain for research and practice. Addiction 2010;105:954-9.

24. Sullivan LE, Tetrault JM, Braithwaite RS, et al. A meta-analysis of the efficacy of nonphysician brief interventions for unhealthy alcohol use: implications for the patient-centered medical home. Am J Addict 2011;20:343-56.

25. HM Government. The Government's Alcohol Strategy. London: Drugs and Alcohol Unit, Home Office, Her Majesty's Government, 2012.

26. Scottish Government. Chief executive's letter, 2007. Guidance on HEAT Targets for NHS Boards. Edinburgh: Scottish Government, 2007.

27. National Institute for Health and Care Excellence. Alcohol use disorders: preventing harmful drinking. London: National Institute for Health and Care Excellence, 2010.

28. World Health Organisation. Global strategy to reduce the harmful use of alcohol. Italy: World Health Organisation, 2010.

29. Liberati A, Altman DG, Tetzlaff J, et al. The PRISMA statement for reporting systematic reviews and meta-analyses of studies that evaluate healthcare interventions: explanation and elaboration. BMJ 2009;339:b2700.

30. Higgins JPT, Green S, eds. Cochrane handbook for systematic reviews of interventions. Version 5.1.0. The Cochrane Collaboration, 2011.

31. Van den Noortgate W, López-López J, Marín-Martínez F, et al. Meta-analysis of multiple outcomes: a multilevel approach. Behav Res 2015;47:1274-94.

32. Viechtbauer W. Conducting meta-analyses in $\mathrm{R}$ with the metafor package. J Stat Softw 2010;36:1-48.

33. Cheung MW. Modeling dependent effect sizes with three-level meta-analyses: a structural equation modeling approach. Psychol Methods 2014;19:211-29.

34. Borenstein M, Hedges LV, Higgins JPT, et al. Introduction to metaanalysis. Chichester, West Sussex: John Wiley and Sons, 2009.

35. Fleming MF, Barry KL, Manwell LB, et al. Brief physician advice for problem alcohol drinkers. A randomized controlled trial in community-based primary care practices. JAMA 1997;277: 1039-45.

36. Grossberg PM, Brown DD, Fleming MF. Brief physician advice for high-risk drinking among young adults. Ann Fam Med 2004;2:474-80.

37. Manwell LB, Fleming MF, Mundt MP, et al. Treatment of problem alcohol use in women of childbearing age: results of a brief intervention trial. Alcohol Clin Exp Res 2000;24:1517-24.

38. Seppä K. Intervention in alcohol abuse among macrocytic patients in general practice. Scand J Prim Health Care 1992;10:217-22.

39. Aalto $M$, Saksanen $R$, Laine $P$, et al. Brief intervention for female heavy drinkers in routine general practice: a 3-year randomized, controlled study. Alcohol Clin Exp Res 2000;24:1680-6.

40. Aalto $M$, Seppa K, Mattila $P$, et al. Brief intervention for male heavy drinkers in routine general practice: a three-year randomized controlled study. Alcohol Alcohol 2001;36:224-30

41. Anderson $\mathrm{P}$, Scott $\mathrm{E}$. The effect of general practitioners' advice to heavy drinking men. Br J Addict 1992;87:891-900. 
42. Antti-Poika I, Karaharju E, Roine R, et al. Intervention of heavy drinking - a prospective and controlled study of 438 consecutive injured male patients. Alcohol Alcohol 1988;23:115-21.

43. Baer JS, Kivlahan DR, Blume AW, et al. Brief intervention for heavy-drinking college students: 4-year follow-up and natural history. Am J Public Health 2001;91:1310-16.

44. Beich A, Gannik D, Saelan H, et al. Screening and brief intervention targeting risky drinkers in Danish general practice-a pragmatic controlled trial. Alcohol Alcohol 2007;42:593-603.

45. Bernstein J, Heeren T, Edward E, et al. A brief motivational interview in a pediatric emergency department, plus 10-day telephone follow-up, increases attempts to quit drinking among youth and young adults who screen positive for problematic drinking. Acad Emerg Med 2010;17:890-902.

46. Butler LH, Correia CJ. Brief alcohol intervention with college student drinkers: face-to-face versus computerized feedback. Psychol Addict Behav 2009;23:163-7.

47. Carey KB, Carey MP, Maisto SA, et al. Brief motivational interventions for heavy college drinkers: a randomized controlled trial. J Consult Clin Psychol 2006;74:943-54.

48. Cherpitel CJ, Korcha RA, Moskalewicz J, et al. Screening, brief intervention, and referral to treatment (SBIRT): 12-month outcomes of a randomized controlled clinical trial in a Polish emergency department. Alcohol Clin Exp Res 2010;34:1922-8.

49. Chick J, Lloyd G, Crombie E. Counselling problem drinkers in medical wards: a controlled study. BMJ (Clin Res Ed) 1985;290:965-7.

50. Cordoba R, Delgado M, Pico V, et al. Effectiveness of brief intervention on non-dependent alcohol drinkers (EBIAL): a Spanish multi-centre study. Fam Pract 1998;15:562-8.

51. Crawford MJ, Patton R, Touquet R, et al. Screening and referral for brief intervention of alcohol-misusing patients in an emergency department: a pragmatic randomised controlled trial. Lancet 2004;364:1334-9.

52. Crawford MJ, Sanatinia R, Barrett B, et al. The clinical effectiveness and cost-effectiveness of brief intervention for excessive alcohol consumption among people attending sexual health clinics: a randomised controlled trial (SHEAR). Health Technol Assess 2014;18:1-48.

53. Curry SJ, Ludman EJ, Grothaus LC, et al. A randomized trial of a brief primary-care-based intervention for reducing at-risk drinking practices. Health Psychol 2003;22:156-65.

54. Daeppen J, Gaume J, Bady P, et al. Brief alcohol intervention and alcohol assessment do not influence alcohol use in injured patients treated in the emergency department: a randomized controlled clinical trial [corrected] [published erratum appears in ADDICTION 2007 Dec;102(12):1995]. Addiction 2007:102:1224-33.

55. Daeppen JB, Bertholet N, Gaume J, et al. Efficacy of brief motivational intervention in reducing binge drinking in young men: a randomized controlled trial. Drug Alcohol Depend 2011;113:69-75.

56. Drummond C, Deluca P, Coulton S, et al. The effectiveness of alcohol screening and brief intervention in emergency departments: a multicentre pragmatic cluster randomized controlled trial. PLOS ONE 2014;9:e99463.

57. Field CA, Caetano R, Harris TR, et al. Ethnic differences in drinking outcomes following a brief alcohol intervention in the trauma care setting. Addiction 2010;105:62-73.

58. Fleming M, Manwell LB. Brief intervention in primary care settings: a primary treatment method for at-risk, problem, and dependent drinkers. Alcohol Res 1999;23:128-37.

59. Fleming MF, Balousek SL, Grossberg PM, et al. Brief physician advice for heavy drinking college students: a randomized controlled trial in college health clinics. J Stud Alcohol Drugs 2010;71:23-31.

60. Freyer-Adam J, Coder B, Baumeister SE, et al. Brief alcohol intervention for general hospital inpatients: a randomized controlled trial. Drug Alcohol Depend 2008;93:233-43.

61. Gaume J, Gmel G, Faouzi M, et al. Is brief motivational intervention effective in reducing alcohol use among young men voluntarily receiving it? A randomized controlled trial. Alcohol Clin Exp Res 2011;35:1822-30.

62. Gaume J, Magill M, Longabaugh R, et al. Influence of counselor characteristics and behaviors on the efficacy of a brief motivational intervention for heavy drinking in young men-a randomized controlled trial. Alcohol Clin Exp Res 2014;38:2138-47.

63. Gentilello LM, Rivara FP, Donovan DM, et al. Alcohol interventions in a trauma center as a means of reducing the risk of injury recurrence. Ann Surg 1999;230:473-80; discussion 80-3.

64. Hansen AB, Becker U, Nielsen AS, et al. Internet-based brief personalized feedback intervention in a non-treatment-seeking population of adult heavy drinkers: a randomized controlled trial. J Med Internet Res 2012;14:e98.

65. Heather N, Campion PD, Neville RG, et al. Evaluation of a controlled drinking minimal intervention for problem drinkers in general practice (the DRAMS scheme). J R Coll Gen Pract 1987;37:358-63.

66. Holloway AS, Watson HE, Arthur AJ, et al. The effect of brief interventions on alcohol consumption among heavy drinkers in a general hospital setting. Addiction 2007;102:1762-70.

67. Ingersoll KS, Ceperich SD, Hettema JE, et al. Preconceptional motivational interviewing interventions to reduce alcohol-exposed pregnancy risk. J Subst Abuse Treat 2013;44:407-16.

68. Juárez $\mathrm{P}$, Walters $\mathrm{ST}$, Daugherty $\mathrm{M}$, et al. A randomized trial of motivational interviewing and feedback with heavy drinking college students. J Drug Educ 2006;36:233-46.

69. Kulesza M, Apperson M, Larimer ME, et al. Brief alcohol intervention for college drinkers: how brief is? Addict Behav 2010;35:730-3.

70. Kulesza M, McVay MA, Larimer ME, et al. A randomized clinical trial comparing the efficacy of two active conditions of a brief intervention for heavy college drinkers. Addict Behav 2013;38:2094-101.

71. Larimer ME, Turner AP, Anderson BK, et al. Evaluating a brief alcohol intervention with fraternities. J Stud Alcohol $2001 ; 62: 370-80$.

72. Liu SI, Wu SI, Chen SC, et al. Randomized controlled trial of a brief intervention for unhealthy alcohol use in hospitalized Taiwanese men. Addiction 2011;106:928-40.

73. Lock CA, Kaner E, Heather N, et al. Effectiveness of nurse-led brief alcohol intervention: a cluster randomized controlled trial. J Adv Nurs 2006;54:426-39.

74. Maisto SA, Conigliaro J, McNeil M, et al. Effects of two types of brief intervention and readiness to change on alcohol use in hazardous drinkers. J Stud Alcohol 2001;62:605-14.

75. Murphy JG, Duchnick JJ, Vuchinich RE, et al. Relative efficacy of a brief motivational intervention for college student drinkers. Psychol Addict Behav 2001;15:373-9.

76. Noknoy S, Rangsin R, Saengcharnchai P, et al. RCT of effectiveness of motivational enhancement therapy delivered by nurses for hazardous drinkers in primary care units in Thailand. Alcohol Alcohol 2010;45:263-70.

77. Richmond R, Heather N, Wodak A, et al. Controlled evaluation of a general practice-based brief intervention for excessive drinking. Addiction 1995;90:119-32.

78. Rubio G, Jiménez-Arriero MA, Martínez I, et al. Efficacy of physician-delivered brief counseling intervention for binge drinkers. Am J Med 2010;123:72-8.

79. Rubio DM, Day NL, Conigliaro J, et al. Brief motivational enhancement intervention to prevent or reduce postpartum alcohol use: a single-blinded, randomized controlled effectiveness trial. J Subst Abuse Treat 2014;46:382-9.

80. Saitz R, Palfai TP, Cheng DM, et al. Brief intervention for medical inpatients with unhealthy alcohol use: a randomized, controlled trial. Ann Intern Med 2007;146:167-76.

81. Schaus JF, Sole ML, McCoy TP, et al. Alcohol screening and brief intervention in a college student health center: a randomized controlled trial. (College drinking: new research from the National Institute on Alcohol Abuse and Alcoholism's Rapid Response to College Drinking Problems initiative.). J Stud Alcohol Drugs 2009;70:131-41.

82. Senft RA, Polen MR, Freeborn DK, et al. Brief intervention in a primary care setting for hazardous drinkers. Am J Prev Med 1997;13:464-70.

83. Shiles CJ, Canning UP, Kennell-Webb SA, et al. Randomised controlled trial of a brief alcohol intervention in a general hospital setting. Trials 2013;14:345

84. Smith AJ, Hodgson RJ, Bridgeman $\mathrm{K}$, et al. A randomized controlled trial of a brief intervention after alcohol-related facial injury. Addiction 2003;98:43-52

85. Wagener TL, Leffingwell TR, Mignogna J, et al. Randomized trial comparing computer-delivered and face-to-face personalized feedback interventions for high-risk drinking among college students. J Subst Abuse Treat 2012;43:260-7.

86. Walters ST, Vader AM, Harris TR, et al. Dismantling motivational interviewing and feedback for college drinkers: a randomized clinical trial. J Consult Clin Psychol 2009;77:64-73.

87. Watt K, Shepherd J, Newcombe R. Drunk and dangerous: a randomised controlled trial of alcohol brief intervention for violent offenders. J Exp Criminol 2008;4:1-19.

88. Saitz R, Svikis D, D'Onofrio G, et al. Challenges applying alcohol brief intervention in diverse practice settings: populations, outcomes, and costs. Alcohol Clin Exp Res 2006;30:332-8. 
89. Brooker C, Peters J, McCabe C, et al. The views of nurses to the conduct of a randomised controlled trial of problem drinkers in an accident and emergency department. Int J Nurs Stud 1999;36:33-9.

90. Desy PM, Perhats C. Alcohol screening, brief intervention, and referral in the emergency department: an implementation study. J Emerg Nurs 2008;34:11-19.

91. Rodriguez-Martos A, Castellano Y, Salmeron JM, et al. Simple advice for injured hazardous drinkers: an implementation study. Alcohol Alcohol 2007;42:430-5.

92. Lock CA. Screening and brief alcohol interventions: what, why, who, where and when? A review of the literature. J Subst Use 2004;9:91-101.

93. Werch CE, Owen DM, Carlson JM, et al. One-year follow-up results of the STARS for Families alcohol prevention program. Health Educ Res 2003;18:74-87.

94. Johansson K, Bendtsen P, Akerlind I. Early intervention for problem drinkers: readiness to participate among general practitioners and nurses in Swedish primary health care. Alcohol Alcohol 2002;37:38-42.
95. Lock CA, Kaner EF. Implementation of brief alcohol interventions by nurses in primary care: do non-clinical factors influence practice? Fam Pract 2004;21:270-5.

96. Owens L, Gilmore IT, Pirmohamed M. General practice nurses knowledge of alcohol use and misuse: a questionnaire survey. Alcohol Alcohol 2000;35:259-62.

97. Kaner E, Bland M, Cassidy $\mathrm{P}$, et al. Effectiveness of screening and brief alcohol intervention in primary care (SIPS trial): pragmatic cluster randomised controlled trial. BMJ 2013;346: e8501.

98. Newbury-Birch D, Coulton S, Bland M, et al. Alcohol screening and brief interventions for offenders in the probation setting (SIPS Trial): a pragmatic multicentre cluster randomized controlled trial. Alcohol Alcohol 2014;49:540-8.

99. Marsh HW, Bornmann L, Mutz R, et al. Gender effects in the peer reviews of grant proposals: a comprehensive meta-analysis comparing traditional and multilevel approaches. Rev Educ Res 2009;79:1290-326. 